\title{
EXISTENCE AND CONTINUATION OF SOLUTIONS FOR A NONLINEAR NEUMANN PROBLEM
}

\author{
KRZYSZTOF MUCHEWICZ ${ }^{\dagger}$ AND SŁAWOMIR RYBICKI ${ }^{\ddagger}$
}

\begin{abstract}
In this article we study the existence, continuation and bifurcation from infinity of nonconstant solutions for a nonlinear Neumann problem. We apply the LeraySchauder degree and the degree for $S O(2)$-equivariant gradient operators defined by the second author in [21.
\end{abstract}

\section{INTRODUCTION}

Consider the following nonlinear Neumann problem

$$
\left\{\begin{aligned}
-\Delta u & =f(u) & & \text { in } \Omega, \\
\frac{\partial u}{\partial \nu} & =0 & & \text { on } \partial \Omega,
\end{aligned}\right.
$$

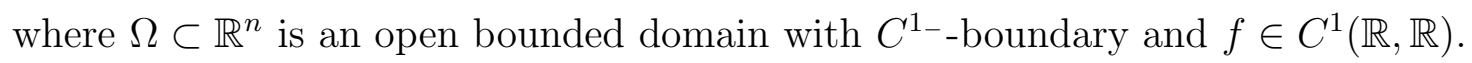

The existence and multiplicity of weak solutions of problem (1.1) has been studied by many authors, see for instance Hirano and Wan [12], Ko [14, Li [15], Li and Li [16], Pomponio [19], Tang [24], Tang and $\mathrm{Wu}$ [25], 26], Vanella[27] and references therein.

Usually weak solutions of system (1.1) are considered as critical points of a functional $\Phi \in C^{2}\left(H^{1}(\Omega), \mathbb{R}\right)$. The authors apply tools of the critical point theory, like the Morse theory, the Conley index technique and the mountain pass theorem, to obtain results.

Solutions of problem (1.1) with special properties focused attention of many authors. The multipeak solutions of problem (1.1) has been extensively studied among the others by Grossi, Pistoia and Wei [11], Dancer and Yan [5]-77, Wang [30] and Yan [33].

A multiplicity of solutions of problem (1.1) in the presence of symmetries of a compact Lie group has been studied among the others by Byeon [4], Vanella [28, Wang [29]- 31].

The aim of this article is to study connected sets of solutions of problem (1.1).

The first goal of this article is to prove the sufficient conditions for the existence of solutions of problem (1.1).

Date: July 31, 2018.

2000 Mathematics Subject Classification. Primary: 35J65; Secondary: 35J25.

Key words and phrases. Leray-Schauder degree, degree for $\mathrm{SO}(2)$-equivariant gradient maps, Neumann boundary value problem, bifurcation of solutions, continuation of solutions.

$\dagger$ Research sponsored by the Doctoral Program in Mathematics at the Nicolaus Copernicus University, Torun, Poland.

${ }^{\ddagger}$ Partially supported by the Ministry Education and Science, Poland, under grant 1 PO3A 00927. 
Let $\sigma(-\Delta ; \Omega)=\left\{0=\lambda_{1}<\lambda_{2}<\ldots\right\}$ denote the set of eigenvalues of the following eigenvalue problem

$$
\left\{\begin{array}{rlr}
-\Delta u & =\lambda u & \text { in } \Omega, \\
\frac{\partial u}{\partial \nu}=0 & \text { on } \partial \Omega,
\end{array}\right.
$$

and let $\mathbb{V}_{-\Delta}\left(\lambda_{i}\right)$ be the eigenspace of the Laplace operator $-\Delta$ corresponding to the eigenvalue $\lambda_{i} \in \sigma(-\Delta ; \Omega)$.

We assume that $f$ is asymptotically linear i.e. $f(x)=f^{\prime}(\infty) x+o(|x|)$, as $|x| \rightarrow \infty$ and that $Z=f^{-1}(0)$ is finite.

In our theorems we put assumptions on $f^{\prime}(z)$, where $z \in Z \cup\{\infty\}$. We emphasize that we also treat problems with resonance at constant solutions and at infinity i.e. it can happen that $f^{\prime}(z) \in \sigma(-\Delta ; \Omega)$ for some $z \in Z \cup\{\infty\}$.

The second goal of this article is to prove the sufficient conditions for continuation of solutions of the following problem

$$
\left\{\begin{aligned}
-\Delta u & =f(u, \lambda) & & \text { in } \Omega, \\
\frac{\partial u}{\partial \nu} & =0 & & \text { on } \partial \Omega,
\end{aligned}\right.
$$

where $\Omega \subset \mathbb{R}^{n}$ is an open bounded domain with $C^{1-}$-boundary and $f \in C^{1}(\mathbb{R} \times \mathbb{R}, \mathbb{R})$.

The third goal of this paper is to study global bifurcations from infinity of solutions of problem (1.3).

It is worth in pointing out that application of classical invariants like the Conley index technique and the Morse theory does not ensure the existence of closed connected sets of critical points of variational problems, see Ambrosetti [2], Böhme [3], Ize [13], Marino [17, Takens [23] for examples and discussion.

In other words one can not apply these invariants in order to prove continuation and global bifurcation of solutions of problem (1.3).

Since the gradient $\nabla \Phi \in C^{1}\left(H^{1}(\Omega), H^{1}(\Omega)\right)$ is of the form compact perturbation of the identity, we apply the Leray-Schauder degree and the degree for $S O(2)$-equivariant gradient maps to the study of critical points (critical $S O(2)$-orbits) of the functional $\Phi$.

The choice of the Leray-Schauder degree and the degree for $S O(2)$-equivariant gradient maps seems to be the best adapted to our theory.

After this introduction our article is organized as follows.

Since the degree for $S O(2)$-equivariant gradient maps is not widely known, in Section 2 we have summarized without proofs the relevant material on this invariant, thus making our exposition as self-contained as possible.

In Section 3 we have studied problem (1.2). In Lemma 3.1 we have derived a formula for the Leray-Schauder degree of the gradient $\nabla_{u} \Psi \in C^{1}\left(H^{1}(\Omega) \times \mathbb{R}, H^{1}(\Omega)\right)$ of a functional $\Psi \in C^{2}\left(H^{1}(\Omega) \times \mathbb{R}, \mathbb{R}\right)$ associated with problem (1.2). Suppose now that $\mathbb{R}^{n}$ is an orthogonal $S O(2)$-representation and that $\Omega \subset \mathbb{R}^{n}$ is $S O(2)$-invariant. Under these assumptions $H^{1}(\Omega)$ is an orthogonal $S O(2)$-representation, the functional $\Psi$ is $S O(2)$-invariant and its gradient $\nabla_{u} \Psi$ is $S O(2)$-equivariant. In Lemma 3.2 we have proved a formula for the degree for $S O(2)$-equivariant gradient maps of $\nabla_{u} \Psi$. 
In Section 4 our main results are stated and proved.

Subsection 4.1 is devoted to the study of the existence of nonconstant solutions of problem (1.1). In Theorems 4.1.14.1.4 we consider non-degenerate case i.e. we assume that $f^{\prime}(z) \notin \sigma(-\Delta ; \Omega)$ for every $z \in Z \cup\{\infty\}$. These theorems ensure the existence of at least one nonconstant solution of problem (1.1). Notice that in Theorems 4.1.2 4.1.4 we have assumed that domain $\Omega$ is $S O(2)$-invariant.

We emphasize that in the proofs of Theorems 4.1.2 4.1.4 the degree for $S O(2)$-equivariant gradient maps can not be replaced with the Leray-Schauder degree, see Remark 4.1.3. Additionally, in Theorem 4.1.5 we have proved the existence of at least one nonconstant solution of problem (1.1) in a degenerate case.

In Subsection 4.2 we have studied continuation of nonconstant solutions of problem (1.3). In Theorems 4.2.1, 4.2.2, 4.2.3 we have formulated sufficient conditions for the existence of closed connected sets of solutions of problem (1.3) emanating from a fixed level $\lambda \in \mathbb{R}$.

In Subsection 4.3 we have studied global bifurcations from infinity of nonconstant solutions of problem (1.3). Theorems 4.3.1, 4.3.2 are the main theorems of this section.

In Section 5 we illustrate the main results of this paper. Namely, we consider problem (1.1) with $\Omega=B^{2}$ and $\Omega=(0,1) \times B^{2}$.

\section{Preliminaries}

In this section, for the convenience of the reader, we remind the main properties of the degree for $S O(2)$-equivariant gradient maps defined in [21]. This degree will be denoted briefly by $\nabla_{S O(2)}-$ deg.

Denote by $\Upsilon(S O(2))$ the set of closed subgroups of the group $S O(2)$ i.e. $\Upsilon(S O(2))=$ $\left\{S O(2), \mathbb{Z}_{1}, \mathbb{Z}_{2}, \ldots, \mathbb{Z}_{k}, \ldots\right\}$.

Put $U(S O(2))=\mathbb{Z} \oplus\left(\bigoplus_{k=1}^{\infty} \mathbb{Z}\right)$ and define actions

$$
\begin{gathered}
+, \star: U(S O(2)) \times U(S O(2)) \rightarrow U(S O(2)), \\
\cdot: \mathbb{Z} \times U(S O(2)) \rightarrow U(S O(2)),
\end{gathered}
$$

as follows

$$
\begin{aligned}
\alpha+\beta & =\left(\alpha_{0}+\beta_{0}, \alpha_{1}+\beta_{1}, \ldots, \alpha_{k}+\beta_{k}, \ldots\right), \\
\alpha \star \beta & =\left(\alpha_{0} \cdot \beta_{0}, \alpha_{0} \cdot \beta_{1}+\beta_{0} \cdot \alpha_{1}, \ldots, \alpha_{0} \cdot \beta_{k}+\beta_{0} \cdot \alpha_{k}, \ldots\right), \\
\gamma \cdot \alpha & =\left(\gamma \cdot \alpha_{0}, \gamma \cdot \alpha_{1}, \ldots, \gamma \cdot \alpha_{k}, \ldots\right),
\end{aligned}
$$

where $\alpha=\left(\alpha_{0}, \alpha_{1}, \ldots, \alpha_{k}, \ldots\right), \beta=\left(\beta_{0}, \beta_{1}, \ldots, \beta_{k}, \ldots\right) \in U(S O(2))$ and $\gamma \in \mathbb{Z}$. It is easy to check that $(U(S O(2)),+, \star)$ is a commutative ring with the unit $\mathbb{I}=(1,0, \ldots) \in$ $U(S O(2))$ and the trivial element $\Theta=(0,0, \ldots) \in U(S O(2))$.

The ring $(U(S O(2)),+, \star)$ is called the Euler ring of the group $S O(2)$.

Remark 2.1. Notice that $\alpha=\left(\alpha_{0}, \alpha_{1}, \ldots, \alpha_{k}, \ldots\right) \in U(S O(2))$ is invertible iff $\alpha_{0}= \pm 1$. 
For a definition of the Euler ring $U(G)$, where $G$ is any compact Lie group, we refer the reader to [8].

If $\delta_{1}, \ldots, \delta_{q} \in U(S O(2))$, then we write $\prod_{j=1}^{q} \delta_{j}$ for $\delta_{1} \star \ldots \star \delta_{q}$. Moreover, it is understood that $\prod_{j \in \emptyset} \delta_{j}=\mathbb{I} \in U(S O(2))$.

Let $\mathbb{V}$ be a real, finite-dimensional and orthogonal $S O(2)$-representation. If $v \in \mathbb{V}$, then the subgroup $S O(2)_{v}=\{g \in S O(2): g \cdot v=v\}$ is said to be the isotropy group of $v \in \mathbb{V}$. Let $\Omega \subset \mathbb{V}$ be an open, bounded and $S O(2)$-invariant subset and let $H \in \Upsilon(S O(2))$. Then we define

- $\Omega^{H}=\left\{v \in \Omega: H \subset S O(2)_{v}\right\}=\{v \in \Omega: g v=v \forall g \in H\}$,

- $\Omega_{H}=\left\{v \in \Omega: H=S O(2)_{v}\right\}$.

Fix $k \in \mathbb{N}$ and set

- $C_{S O(2)}^{k}(\mathbb{V}, \mathbb{R})=\left\{f \in C^{k}(\mathbb{V}, \mathbb{R}): f\right.$ is $S O(2)$-invariant $\}$,

- $C_{S O(2)}^{k-1}(\mathbb{V}, \mathbb{V})=\left\{f \in C^{k-1}(\mathbb{V}, \mathbb{V}): f\right.$ is $S O(2)$-equivariant $\}$.

Let $f \in C_{S O(2)}^{1}(\mathbb{V}, \mathbb{R})$. Since $\mathbb{V}$ is an orthogonal $S O(2)$-representation, the gradient $\nabla f \in$ $C_{S O(2)}^{0}(\mathbb{V}, \mathbb{V})$. If $H \in \Upsilon(S O(2))$ is a closed subgroup, then $\mathbb{V}^{H}$ is a finite-dimensional $S O(2)$-representation and $(\nabla f)^{H}=\nabla\left(f_{\mid \mathbb{V} H}\right): \mathbb{V}^{H} \rightarrow \mathbb{V}^{H}$ is well-defined $S O(2)$-equivariant gradient map. Choose an open, bounded and $S O(2)$-invariant subset $\Omega \subset \mathbb{V}$ such that $(\nabla f)^{-1}(0) \cap \partial \Omega=\emptyset$. Under these assumptions we have defined in 21] the degree for $S O(2)$-equivariant gradient maps $\nabla_{S O(2)}-\operatorname{deg}(\nabla f, \Omega) \in U(S O(2))$ with coordinates

$$
\begin{gathered}
\nabla_{S O(2)}-\operatorname{deg}(\nabla f, \Omega)= \\
=\left(\nabla_{S O(2)}-\operatorname{deg}_{S O(2)}(\nabla f, \Omega), \nabla_{S O(2)}-\operatorname{deg}_{\mathbb{Z}_{1}}(\nabla f, \Omega), \ldots, \nabla_{S O(2)}-\operatorname{deg}_{\mathbb{Z}_{k}}(\nabla f, \Omega), \ldots\right) .
\end{gathered}
$$

Remark 2.2. To define the degree for $S O(2)$-equivariant gradient maps of $\nabla f_{0}$ we choose (in a homotopy class of the $S O(2)$-equivariant gradient map $\nabla f_{0}$ ) a sufficiently good $S O(2)$-equivariant gradient map $\nabla f_{1}$ and define this degree for $\nabla f_{1}$. The definition does not depend on the choice of the map $\nabla f_{1}$. Roughly speaking the main steps of the definition of the degree for $S O(2)$-equivariant gradient maps of $\nabla f_{0}:(\operatorname{cl}(\Omega), \partial \Omega) \rightarrow(\mathbb{V}, \mathbb{V} \backslash\{0\})$ are the following:

Step 1. There is a potential $f \in C_{S O(2)}^{1}(\mathbb{V} \times[0,1], \mathbb{R})$ such that

(a1) $\left(\nabla_{v} f\right)^{-1}(0) \cap(\partial \Omega \times[0,1])=\emptyset$,

(a2) $\nabla_{v} f(\cdot, 0)=\nabla f_{0}(\cdot)$,

(a3) $\nabla_{v} f_{1} \in C_{S O(2)}^{1}(\mathbb{V}, \mathbb{V})$, where we abbreviate $\nabla_{v} f(\cdot, 1)$ to $\nabla_{v} f_{1}$,

(a4) $\left(\nabla_{v} f_{1}\right)^{-1}(0) \cap \Omega^{S O(2)}=\left\{v_{1}, \ldots, v_{p}\right\}$ and

(i) $\operatorname{det} \nabla_{v v}^{2} f_{1}\left(v_{j}\right) \neq 0$, for all $j=1, \ldots, p$,

(ii) $\nabla_{v v}^{2} f_{1}\left(v_{j}\right)=\left[\begin{array}{cc}\nabla_{v v}^{2}\left(f_{1}^{S O(2)}\right)\left(v_{j}\right) & 0 \\ 0 & I d\end{array}\right]$ :

$\mathbb{V}^{S O}(2)$

$\mathbb{V}^{S O(2)}$

for all $j=1, \ldots, p$,

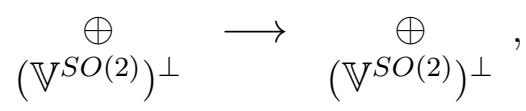


(a5) $\left(\nabla_{v} f_{1}\right)^{-1}(0) \cap\left(\Omega \backslash \Omega^{S O(2)}\right)=\left\{S O(2) w_{1}, \ldots, S O(2) w_{q}\right\}$ and

(i) $\operatorname{dim} \operatorname{ker} \nabla_{v v}^{2} f_{1}\left(w_{j}\right)=1$, for all $j=1, \ldots, q$,

(ii)

$$
\begin{aligned}
& \nabla_{v v}^{2} f_{1}\left(w_{j}\right)=\left[\begin{array}{ccc}
0 & 0 & 0 \\
0 & Q_{j} & 0 \\
0 & 0 & I d
\end{array}\right]: \\
& T_{w_{j}}\left(S O(2) w_{j}\right) \quad T_{w_{j}}\left(S O(2) w_{j}\right) \\
& \oplus \quad \oplus \\
& T_{w_{j}}\left(\mathbb{V}_{S O(2)_{w_{j}}}\right) \ominus T_{w_{j}}\left(S O(2) w_{j}\right) \longrightarrow T_{w_{j}}\left(\mathbb{V}_{S O(2)_{w_{j}}}\right) \ominus T_{w_{j}}\left(S O(2) w_{j}\right) \\
& \oplus \\
& \left(T_{w_{j}}\left(\mathbb{V}_{S O(2)_{w_{j}}}\right)\right)^{\perp} \\
& \left(T_{w_{j}}\left(\mathbb{V}_{S O(2)_{w_{j}}}\right)\right)^{\perp}
\end{aligned}
$$

for all $j=1, \ldots, q$.

Step 2. The first coordinate of the degree for $S O(2)$-equivariant gradient maps is defined by $\nabla_{S O(2)}-\operatorname{deg}_{S O(2)}\left(\nabla f_{0}, \Omega\right)=\sum_{j=1}^{p} \operatorname{sign} \operatorname{det} \nabla_{v v}^{2}\left(f_{1}^{S O(2)}\right)\left(v_{j}\right)$. In other words since $\nabla\left(f_{1}^{S O(2)}\right)=\left(\nabla f_{1}\right)^{S O(2)}$, we obtain

$$
\nabla_{S O(2)}-\operatorname{deg}_{S O(2)}\left(\nabla f_{0}, \Omega\right)=\operatorname{deg}_{\mathrm{B}}\left(\left(\nabla f_{1}\right)^{S O(2)}, \Omega^{S O(2)}, 0\right),
$$

where $\operatorname{deg}_{\mathrm{B}}$ denotes the Brouwer degree.

Step 3. Fix $k \in \mathbb{N}$ and define

$$
\nabla_{S O(2)}-\operatorname{deg}_{\mathbb{Z}_{k}}\left(\nabla f_{0}, \Omega\right)=\sum_{\left\{j \in\{1, \ldots, q\}: S O(2)_{w_{j}}=\mathbb{Z}_{k}\right\}} \operatorname{sign} \operatorname{det} Q_{j},
$$

Notice that since

$$
\operatorname{deg}_{\mathrm{B}}\left(\left(\nabla f_{1}\right)^{S O(2)}, \Omega^{S O(2)}, 0\right)=\operatorname{deg}_{\mathrm{B}}\left(\nabla f_{1}, \Omega, 0\right) \text { and } \operatorname{deg}_{\mathrm{B}}\left(\nabla f_{1}, \Omega, 0\right)=\operatorname{deg}_{\mathrm{B}}\left(\nabla f_{0}, \Omega, 0\right)
$$

(see [20]), directly by the Step 2 . we obtain $\nabla_{S O(2)}-\operatorname{deg}_{S O(2)}\left(\nabla f_{0}, \Omega\right)=\operatorname{deg}_{\mathrm{B}}\left(\nabla f_{0}, \Omega, 0\right)$. Moreover, immediately from the Step 3. we obtain that if $k \in \mathbb{N}$ and $S O(2)_{v} \neq \mathbb{Z}_{k}$ for every $v \in \Omega$, then $\nabla_{S O(2)}-\operatorname{deg}_{\mathbb{Z}_{k}}\left(\nabla f_{0}, \Omega\right)=0$.

For $\gamma>0$ and $v_{0} \in \mathbb{V}^{S O(2)}$ we put $B_{\gamma}\left(\mathbb{V}, v_{0}\right)=\left\{v \in \mathbb{V}:\left|v-v_{0}\right|<\gamma\right\}$ and $D_{\gamma}\left(\mathbb{V}, v_{0}\right)=$ $\left\{v \in \mathbb{V}:\left|v-v_{0}\right| \leq \gamma\right\}$. For simplicity of notation we put $B_{\gamma}(\mathbb{V})=B_{\gamma}(\mathbb{V}, 0)$ and $D_{\gamma}(\mathbb{V})=D_{\gamma}(\mathbb{V}, 0)$.

In the following theorem we formulate the main properties of the degree for $S O(2)$ equivariant gradient maps.

Theorem 2.1 ([21]). Under the above assumptions the degree for $S O(2)$-equivariant gradient maps has the following properties

(1) if $\nabla_{S O(2)}-\operatorname{deg}(\nabla f, \Omega) \neq \Theta$, then $(\nabla f)^{-1}(0) \cap \Omega \neq \emptyset$,

(2) if $\nabla_{S O(2)}-\operatorname{deg}_{H}(\nabla f, \Omega) \neq 0$, then $(\nabla f)^{-1}(0) \cap \Omega^{H} \neq \emptyset$,

(3) if $\Omega=\Omega_{0} \cup \Omega_{1}$ and $\Omega_{0} \cap \Omega_{1}=\emptyset$, then

$$
\nabla_{S O(2)}-\operatorname{deg}(\nabla f, \Omega)=\nabla_{S O(2)}-\operatorname{deg}\left(\nabla f, \Omega_{0}\right)+\nabla_{S O(2)}-\operatorname{deg}\left(\nabla f, \Omega_{1}\right),
$$


(4) if $\Omega_{0} \subset \Omega$ is an open $S O(2)$-invariant subset and $(\nabla f)^{-1}(0) \cap \Omega \subset \Omega_{0}$, then

$$
\nabla_{S O(2)}-\operatorname{deg}(\nabla f, \Omega)=\nabla_{S O(2)}-\operatorname{deg}\left(\nabla f, \Omega_{0}\right),
$$

$(5)$ if $f \in C_{S O(2)}^{1}(\mathbb{V} \times[0,1], \mathbb{R})$ is such that $\left(\nabla_{v} f\right)^{-1}(0) \cap(\partial \Omega \times[0,1])=\emptyset$, then

$$
\nabla_{S O(2)}-\operatorname{deg}\left(\nabla f_{0}, \Omega\right)=\nabla_{S O(2)}-\operatorname{deg}\left(\nabla f_{1}, \Omega\right),
$$

(6) if $W$ is an orthogonal $S O(2)$-representation, then

$$
\nabla_{S O(2)}-\operatorname{deg}\left((\nabla f, I d), \Omega \times B_{\gamma}(W)\right)=\nabla_{S O(2)}-\operatorname{deg}(\nabla f, \Omega)
$$

(7) if $f \in C_{S O(2)}^{2}(\mathbb{V}, \mathbb{R})$ is such that $\nabla f(0)=0$ and $\nabla^{2} f(0)$ is an $S O(2)$-equivariant self-adjoint isomorphism, then there is $\gamma>0$ such that

$$
\nabla_{S O(2)}-\operatorname{deg}\left(\nabla f, B_{\gamma}(\mathbb{V})\right)=\nabla_{S O(2)}-\operatorname{deg}\left(\nabla^{2} f(0), B_{\gamma}(\mathbb{V})\right) .
$$

Remark 2.3. Directly from the definition of the degree for $S O(2)$-equivariant gradient maps (see [21) it follows that

(1) if $H \in \Upsilon(S O(2))$ is a closed subgroup and $S O(2)_{v} \neq H$, for every $v \in \Omega$, then $\nabla_{S O(2)}-\operatorname{deg}_{H}(\nabla f, \Omega)=0$.

(2) $\nabla_{S O(2)}-\operatorname{deg}_{S O(2)}(\nabla f, \Omega)=\operatorname{deg}_{\mathrm{B}}(\nabla f, \Omega, 0)$, where $\operatorname{deg}_{\mathrm{B}}$ is the Brouwer degree.

Below we formulate product formula for the degree for $S O(2)$-equivariant gradient maps.

Theorem $2.2\left([22)\right.$. Let $\Omega_{i} \subset \mathbb{V}_{i}$ be an open, bounded and $S O(2)$-invariant subset of a finite-dimensional, orthogonal $S O(2)$-representation $\mathbb{V}_{i}$, for $i=1,2$. Let $f_{i} \in C_{S O(2)}^{1}\left(\mathbb{V}_{i}, \mathbb{R}\right)$ be such that $\left(\nabla f_{i}\right)^{-1}(0) \cap \partial \Omega_{i}=\emptyset$, for $i=1,2$. Then

$$
\nabla_{S O(2)}-\operatorname{deg}\left(\left(\nabla f_{1}, \nabla f_{2}\right), \Omega_{1} \times \Omega_{2}\right)=\nabla_{S O(2)}-\operatorname{deg}\left(\nabla f_{1}, \Omega_{1}\right) \star \nabla_{S O(2)}-\operatorname{deg}\left(\nabla f_{2}, \Omega_{2}\right) .
$$

For $k \in \mathbb{N}$ define a map $\rho^{k}: S O(2) \rightarrow G L(2, \mathbb{R})$ as follows

$$
\rho^{k}\left(\left[\begin{array}{rr}
\cos \theta & -\sin \theta \\
\sin \theta & \cos \theta
\end{array}\right]\right)=\left[\begin{array}{rr}
\cos (k \cdot \theta) & -\sin (k \cdot \theta) \\
\sin (k \cdot \theta) & \cos (k \cdot \theta)
\end{array}\right] \quad 0 \leq \theta<2 \cdot \pi .
$$

For $j, k \in \mathbb{N}$ we denote by $\mathbb{R}[j, k]$ the direct sum of $j$ copies of $\left(\mathbb{R}^{2}, \rho^{k}\right)$, we also denote by $\mathbb{R}[j, 0]$ the trivial $j$-dimensional $S O(2)$-representation. We say that two $S O(2)$ representations $\mathbb{V}$ and $\mathbb{W}$ are equivalent if there exists an $S O(2)$-equivariant, linear isomorphism $T: \mathbb{V} \rightarrow \mathbb{W}$. The following classic result gives a complete classification (up to equivalence) of finite-dimensional $S O(2)$-representations (see [1]).

Theorem 2.3 ([1]). If $\mathbb{V}$ is a finite-dimensional $S O(2)$-representation, then there exist finite sequences $\left\{j_{i}\right\},\left\{k_{i}\right\}$ satisfying:

$(*) \quad k_{i} \in\{0\} \cup \mathbb{N}, \quad j_{i} \in \mathbb{N}, \quad 1 \leq i \leq r, k_{1}<k_{2}<\cdots<k_{r}$

such that $\mathbb{V}$ is equivalent to $\bigoplus_{i=1}^{r} \mathbb{R}\left[j_{i}, k_{i}\right]$. Moreover, the equivalence class of $\mathbb{V} \mathbb{V} \approx$ $\left.\bigoplus_{i=1}^{r} \mathbb{R}\left[j_{i}, k_{i}\right]\right)$ is uniquely determined by $\left\{k_{i}\right\},\left\{j_{i}\right\}$ satisfying $(*)$. 
Notice that if $\mathbb{V} \approx \bigoplus_{i=1}^{r} \mathbb{R}\left[j_{i}, k_{i}\right]$ and $k_{1}=0$, then $\mathbb{V}^{S O(2)} \approx \mathbb{R}\left[j_{1}, 0\right]$. An $S O(2)$-representation $\mathbb{V}$ is called nontrivial if $\mathbb{V}^{S O(2)} \neq \mathbb{V}$. Suppose that $j^{\prime} \in \mathbb{N}, k^{\prime} \in \mathbb{N} \cup\{0\}$ and $\mathbb{V} \approx \bigoplus_{i=1}^{r} \mathbb{R}\left[j_{i}, k_{i}\right]$. It is understood that if $\mathbb{R}\left[1, k^{\prime}\right] \not \subset \mathbb{V}$, then $k^{\prime} \neq k_{i}$ for $i=1, \ldots, r$. Moreover, if $k^{\prime} \in \mathbb{N}$, then $\mathbb{V}_{\mathbb{Z}_{k^{\prime}}}=\emptyset$ is equivalent to $k^{\prime} \neq \operatorname{gcd}\left(k_{i_{1}}, \ldots, k_{i_{s}}\right)$ for every $\left\{i_{1}, \ldots, i_{s}\right\} \subset\{1, \ldots, r\}$.

We will denote by $m^{-}(L)$ the Morse index of a symmetric matrix $L$.

To apply successfully any degree theory we need computational formulas for this invariant. Below we show how to compute degree for $S O(2)$-equivariant gradient maps of a linear, self-adjoint, $S O(2)$-equivariant isomorphism.

Lemma 2.1 ([21]). If $\mathbb{V} \approx \mathbb{R}\left[j_{0}, 0\right] \oplus \mathbb{R}\left[j_{1}, k_{1}\right] \oplus \ldots \oplus \mathbb{R}\left[j_{r}, k_{r}\right], L: \mathbb{V} \rightarrow \mathbb{V}$ is a self-adjoint, $S O(2)$-equivariant, linear isomorphism and $\gamma>0$, then

(1) $L=\operatorname{diag}\left(L_{0}, L_{1}, \ldots, L_{r}\right)$

$$
\nabla_{S O(2)}-\operatorname{deg}_{H}\left(L, B_{\gamma}(\mathbb{V})\right)= \begin{cases}(-1)^{m^{-}\left(L_{0}\right)}, & \text { for } H=S O(2), \\ (-1)^{m^{-}\left(L_{0}\right)} \cdot \frac{m^{-}\left(L_{i}\right)}{2}, & \text { for } H=\mathbb{Z}_{k_{i}} \\ 0, & \text { for } H \notin\left\{S O(2), \mathbb{Z}_{k_{1}}, \ldots, \mathbb{Z}_{k_{r}}\right\},\end{cases}
$$

(3) in particular, if $L=-I d$, then

$$
\nabla_{S O(2)}-\operatorname{deg}_{H}\left(-I d, B_{\gamma}(\mathbb{V})\right)= \begin{cases}(-1)^{j_{0}}, & \text { for } H=S O(2) \\ (-1)^{j_{0}} \cdot j_{i}, & \text { for } H=\mathbb{Z}_{k_{i}} \\ 0, & \text { for } H \notin\left\{S O(2), \mathbb{Z}_{k_{1}}, \ldots, \mathbb{Z}_{k_{r}}\right\}\end{cases}
$$

Let $\left(\mathbb{H},\langle\cdot, \cdot\rangle_{\mathbb{H}}\right)$ be an infinite-dimensional, separable Hilbert space which is an orthogonal $S O(2)$-representation and let $C_{S O(2)}^{1}(\mathbb{H}, \mathbb{R})$ denote the set of $S O(2)$-invariant $C^{1}$ functionals. Fix $\Phi \in C_{S O(2)}^{1}(\mathbb{H}, \mathbb{R})$ such that

$$
\nabla \Phi(u)=u-\nabla \eta(u)
$$

where $\nabla \eta: \mathbb{H} \rightarrow \mathbb{H}$ is an $S O(2)$-equivariant compact operator. Let $\mathcal{U} \subset \mathbb{H}$ be an open, bounded and $S O(2)$-invariant set such that $(\nabla \Phi)^{-1}(0) \cap \partial \mathcal{U}=\emptyset$. In this situation $\nabla_{S O(2)}-\operatorname{deg}(I d-\nabla \eta, \mathcal{U}) \in U(S O(2))$ is well-defined, see [21] for details and properties of this degree.

Let $L: \mathbb{H} \rightarrow \mathbb{H}$ be a linear, bounded, self-adjoint, $S O(2)$-equivariant operator with spectrum $\sigma(L)=\left\{\lambda_{i}\right\}$. By $\mathbb{V}_{L}\left(\lambda_{i}\right)$ we will denote eigenspace of $L$ corresponding to the eigenvalue $\lambda_{i}$ and we put $\mu_{L}\left(\lambda_{i}\right)=\operatorname{dim} \mathbb{V}_{L}\left(\lambda_{i}\right)$. In other words $\mu_{L}\left(\lambda_{i}\right)$ is the multiplicity of the eigenvalue $\lambda_{i}$. Since operator $L$ is linear, bounded, self-adjoint, and $S O(2)$-equivariant, $\mathbb{V}_{L}\left(\lambda_{i}\right)$ is a finite-dimensional, orthogonal $S O(2)$-representation.

For $\gamma>0$ and $v_{0} \in \mathbb{H}^{S O(2)}$ put $B_{\gamma}\left(\mathbb{H}, v_{0}\right)=\left\{v \in \mathbb{H}:\left|v-v_{0}\right|<\gamma\right\}$. For simplicity of notation $B_{\gamma}(\mathbb{H})$ stands for $B_{\gamma}(\mathbb{H}, 0)$. 
Combining Theorem 4.5 in [21] with Theorem 2.2 we obtain the following theorem.

Theorem 2.4. Under the above assumptions if $1 \notin \sigma(L)$, then

$$
\nabla_{S O(2)}-\operatorname{deg}\left(I d-L, B_{\gamma}(\mathbb{H})\right)=\prod_{\lambda_{i}>1} \nabla_{S O(2)}-\operatorname{deg}\left(-I d, B_{\gamma}\left(\mathbb{V}_{L}\left(\lambda_{i}\right)\right)\right) \in U(S O(2)) .
$$

It is understood that if $\sigma(L) \cap[1,+\infty)=\emptyset$, then

$$
\nabla_{S O(2)}-\operatorname{deg}\left(I d-L, B_{\gamma}(\mathbb{H})\right)=\mathbb{I} \in U(S O(2)) .
$$

Below we formulate the continuation theorem for $S O(2)$-equivariant gradient operators of the form compact perturbation of the identity. In other words we study continuation of critical orbits of $S O(2)$-invariant $C^{1}$-functionals. The proof of this theorem is standard, but in the proof we have to replace the Leray-Schauder degree with the degree for $S O(2)$ equivariant gradient operators.

Theorem 2.5. Let $\Phi \in C_{S O(2)}^{1}(\mathbb{H} \times \mathbb{R}, \mathbb{R})$ be such that $\nabla_{u} \Phi(u, \lambda)=u-\nabla_{u} \eta(u, \lambda)$, where $\nabla \eta: \mathbb{H} \times \mathbb{R} \rightarrow \mathbb{H}$ is an $S O(2)$-equivariant compact operator. Fix an open, bounded and $S O(2)$-invariant subset $\mathcal{U} \subset \mathbb{H}$ and $\lambda_{0} \in \mathbb{R}$ such that

(1) $\left(\nabla_{u} \Phi\left(\cdot, \lambda_{0}\right)\right)^{-1}(0) \cap \partial \mathcal{U}=\emptyset$

(2) $\nabla_{S O(2)}-\operatorname{deg}\left(\nabla_{u} \Phi\left(\cdot, \lambda_{0}\right), \mathcal{U}\right) \neq \Theta \in U(S O(2))$.

Then there exist continua (closed connected sets) $\mathcal{C}^{ \pm} \subset \mathbb{H} \times \mathbb{R}$, with

$$
\begin{aligned}
& \mathcal{C}^{-} \subset\left(\mathbb{H} \times\left(-\infty, \lambda_{0}\right]\right) \cap\left(\nabla_{u} \Phi\left(\cdot, \lambda_{0}\right)\right)^{-1}(0), \\
& \mathcal{C}^{+} \subset\left(\mathbb{H} \times\left[\lambda_{0},+\infty\right)\right) \cap\left(\nabla_{u} \Phi\left(\cdot, \lambda_{0}\right)\right)^{-1}(0),
\end{aligned}
$$

and for both $\mathcal{C}=\mathcal{C}^{ \pm}$the following statements are valid

(1) $\mathcal{C} \cap\left(\mathcal{U} \times\left\{\lambda_{0}\right\}\right) \neq \emptyset$

(2) either $\mathcal{C}$ is unbounded or else $\mathcal{C} \cap\left((\mathbb{H} \backslash \operatorname{cl}(\mathcal{U})) \times\left\{\lambda_{0}\right\}\right) \neq \emptyset$.

¿From now on let $\Phi \in C_{S O(2)}^{2}(\mathbb{H} \times \mathbb{R}, \mathbb{R})$ be such that $\nabla_{u} \Phi(u, \lambda)=u-\nabla_{u} \eta(u, \lambda)$, where $\nabla \eta: \mathbb{H} \times \mathbb{R} \rightarrow \mathbb{H}$ is an $S O(2)$-equivariant compact operator. Fix $\lambda_{+}>\lambda_{-}$and assume that there exists $\gamma>0$ such that

$$
\left(\nabla \Phi_{u}\left(\cdot, \lambda_{ \pm}\right)\right)^{-1}(0) \subset B_{\gamma}(\mathbb{H}) \times\left\{\lambda_{ \pm}\right\}=\emptyset .
$$

Definition 2.1. An element $\operatorname{BIF}\left(\infty,\left[\lambda_{-}, \lambda_{+}\right]\right) \in U(S O(2))$ defined as follows

$$
\operatorname{BIF}\left(\infty,\left[\lambda_{-}, \lambda_{+}\right]\right)=\nabla_{S O(2)}-\operatorname{deg}\left(\nabla_{u} \Phi\left(\cdot, \lambda_{+}\right), B_{\gamma}(\mathbb{H})\right)-\nabla_{S O(2)}-\operatorname{deg}\left(\nabla_{u} \Phi\left(\cdot, \lambda_{-}\right), B_{\gamma}(\mathbb{H})\right)
$$

is called the bifurcation index at $\left(\infty,\left[\lambda_{-}, \lambda_{+}\right]\right)$.

In the following theorems we have formulated sufficient conditions for the existence of an unbounded closed connected set of critical orbits bifurcating from infinity. Proofs of this theorems can be found in [10].

Theorem 2.6. Take $\Phi$ as above and let $\lambda_{ \pm} \in \mathbb{R}, \gamma>0$ be such that condition (2.5) holds. If $\operatorname{BIF}\left(\infty,\left[\lambda_{-}, \lambda_{+}\right]\right) \neq \Theta \in U(S O(2))$, then there exists an unbounded closed connected component $C$ of $\left(\nabla_{u} \Phi\right)^{-1}(0) \cap\left(\mathbb{H} \times\left[\lambda_{-}, \lambda_{+}\right]\right)$such that $C \cap\left(B_{\gamma}(\mathbb{H}) \times\left\{\lambda_{-}, \lambda_{+}\right\}\right) \neq \emptyset$. 
Let $\Phi$ satisfy the following additional assumption:

$\Phi(u, \lambda)=\frac{1}{2}\langle u, u\rangle_{\mathbb{H}}-\frac{1}{2}\left\langle K_{\infty}(\lambda) u, u\right\rangle_{\mathbb{H}}-\eta_{\infty}(u, \lambda)$, where

(1) $K_{\infty}(\lambda): \mathbb{H} \rightarrow \mathbb{H}$ is a linear $S O(2)$-equivariant self-adjoint operator for every $\lambda \in \mathbb{R}$

(2) the mapping $\mathbb{H} \times \mathbb{R} \ni(u, \lambda) \mapsto K_{\infty}(\lambda) u \in \mathbb{H}$ is compact,

(3) $\nabla_{u} \eta_{\infty}: \mathbb{H} \times \mathbb{R} \rightarrow \mathbb{H}$ is an $S O(2)$-equivariant compact operator such that $\nabla_{u} \eta_{\infty}(u, \lambda)=o(|u|)$, as $|u| \rightarrow \infty$ uniformly on bounded $\lambda$-intervals.

For $\lambda \in \mathbb{R}$ define $\nabla_{u}^{2} \Phi(\infty, \lambda)=I d-K_{\infty}(\lambda)$. Fix arbitrary $\lambda_{0} \in \mathbb{R}$ and assume that ker $\nabla_{u}^{2} \Phi\left(\infty, \lambda_{0}\right) \neq\{0\}$. Choose $\epsilon>0$, define $\lambda_{ \pm}=\lambda_{0} \pm \epsilon$ and assume that the following condition is fulfilled

$$
\left\{\lambda \in\left[\lambda_{-}, \lambda_{+}\right]: \nabla_{u}^{2} \Phi(\infty, \lambda) \text { is not an isomorphism }\right\}=\left\{\lambda_{0}\right\} .
$$

Definition 2.2. We say that an unbounded closed connected set $\mathcal{C}$ meets $\left(\infty, \lambda_{0}\right)$, if for every $\delta, \eta>0$

$$
\mathcal{C} \cap\left\{\left(H^{1}(\Omega) \backslash B_{\gamma}\left(H^{1}(\Omega)\right)\right) \times\left[\lambda_{0}-\delta, \lambda_{0}+\delta\right]\right\} \neq \emptyset .
$$

The following theorem localize points at which closed connected sets of solutions of equation $\nabla_{u} \Phi(u, \lambda)=0$ meet infinity.

Theorem 2.7. Let $\Phi$ be as above. Choose $\epsilon, \gamma>0, \lambda_{0}, \lambda_{ \pm} \in \mathbb{R}$ such that the above conditions are satisfied. If $\operatorname{BIF}\left(\infty,\left[\lambda_{-}, \lambda_{+}\right]\right) \neq \Theta \in U(S O(2))$, then the statement of Theorem 2.6 holds true. Moreover, $\mathcal{C}$ meets $\left(\infty, \lambda_{0}\right)$.

\section{LINEAR EQUATION}

Throughout this section we assume that $\Omega \subset \mathbb{R}^{n}$ is a bounded, open set with $C^{1_{-}}$ boundary. Consider the following eigenvalue problem

$$
\left\{\begin{aligned}
-\Delta u & =\lambda u & & \text { in } \Omega, \\
\frac{\partial u}{\partial \nu} & =0 & & \text { on } \partial \Omega .
\end{aligned}\right.
$$

Denote by $\sigma(-\Delta ; \Omega):=\left\{0=\lambda_{1}<\lambda_{2}<\ldots\right\}$ the set of distinct eigenvalues of problem (3.1). Let $\mathbb{V}_{-\Delta}\left(\lambda_{i}\right)$ be the eigenspace of $-\Delta$ corresponding to the eigenvalue $\lambda_{i} \in$ $\sigma(-\Delta ; \Omega)$. Additionally define

$$
\nu(\lambda)= \begin{cases}\sum_{\lambda_{i}<\lambda} \operatorname{dim} \mathbb{V}_{-\Delta}\left(\lambda_{i}\right) & \text { if } \lambda>0, \\ 0 & \text { if } \lambda \leq 0 .\end{cases}
$$

Solutions of problem (3.1) are in one to one correspondence with critical points of functional $\Psi: H^{1}(\Omega) \times \mathbb{R} \rightarrow \mathbb{R}$ defined by

$$
\Psi(u, \lambda)=\frac{1}{2} \int_{\Omega}|\nabla u|^{2}-\lambda u^{2} d x .
$$

Computing the gradient $\nabla_{u} \Psi: H^{1}(\Omega) \times \mathbb{R} \rightarrow H^{1}(\Omega)$ we obtain

$$
\left\langle\nabla_{u} \Psi(u, \lambda), v\right\rangle_{H^{1}(\Omega)}=\int_{\Omega} \nabla u \nabla v-\lambda u v d x=
$$




$$
=\int_{\Omega} \nabla u \nabla v+u v-u v-\lambda u v d x=\langle u, v\rangle_{H^{1}(\Omega)}-(\lambda+1) \int_{\Omega} u v d x .
$$

According to the Riesz theorem there exists linear bounded operator $\mathcal{K}: H^{1}(\Omega) \rightarrow H^{1}(\Omega)$ given by formula $\langle\mathcal{K} u, v\rangle_{H^{1}(\Omega)}=\int_{\Omega} u v d x$. By definition $\mathcal{K}$ is self adjoint and by the imbedding theorems it is compact. Hence, $\nabla_{u} \Psi(u, \lambda)=u-(\lambda+1) \mathcal{K} u$.

Fix $\lambda_{i} \in \sigma(-\Delta ; \Omega)$ and $u_{i} \in \mathbb{V}_{-\Delta}\left(\lambda_{i}\right)$. Thus $\nabla_{u} \Psi\left(u_{i}, \lambda_{i}\right)=0$ and consequently

$$
\nabla_{u} \Psi\left(u_{i}, \lambda\right)=u_{i}-(\lambda+1) \mathcal{K} u_{i}=u_{i}-\frac{\lambda+1}{\lambda_{i}+1} u_{i}=\frac{\lambda_{i}-\lambda}{\lambda_{i}+1} u_{i} .
$$

By the spectral theorem for compact, self-adjoint operators $H^{1}(\Omega)=\overline{\bigoplus_{i=1}^{\infty} \mathbb{V}_{-\Delta}\left(\lambda_{i}\right)}$. Moreover, for every $u \in H^{1}(\Omega)$ there exists a unique representation $u=\sum_{i=1}^{\infty} u_{i}$ such that $u_{i} \in \mathbb{V}_{-\Delta}\left(\lambda_{i}\right)$ for $i \in \mathbb{N} \cup\{0\}$. Hence by (3.2) we obtain

$$
\nabla_{u} \Psi(u, \lambda)=u-(1+\lambda) \mathcal{K} u=\sum_{i=0}^{\infty}\left(\frac{\lambda_{i}-\lambda}{\lambda_{i}+1}\right) u_{i}
$$

Since $\nabla_{u} \Psi(\cdot, \lambda)$ is a family of operators of the form compact perturbation of the identity, one can apply the Leray-Schauder $\operatorname{deg}_{\mathrm{LS}}$ degree to $\nabla_{u} \Psi(\cdot, \lambda)$.

The standard proof of the following lemma is omitted.

Lemma 3.1. Fix $\lambda \notin \sigma(-\Delta ; \Omega)$ and $\gamma>0$. Then

$$
\operatorname{deg}_{L S}\left(\nabla_{u} \Psi(\cdot, \lambda), B_{\gamma}\left(H^{1}(\Omega)\right), 0\right)=(-1)^{\nu(\lambda)} .
$$

Remark 3.1. If $\lambda \in(0,+\infty) \backslash \sigma(-\Delta ; \Omega)$, then

$$
\operatorname{deg}_{\mathrm{LS}}\left(\nabla_{u} \Psi(\cdot, \lambda), B_{\gamma}\left(H^{1}(\Omega)\right), 0\right)=\prod_{\lambda_{i}<\lambda} \operatorname{deg}_{\mathrm{LS}}\left(-I d, B_{\gamma}\left(\mathbb{V}_{-\Delta}\left(\lambda_{i}\right)\right), 0\right) \in\{ \pm 1\} .
$$

If $\lambda<0$, then it is understood that $\operatorname{deg}_{\mathrm{LS}}\left(\nabla_{u} \Psi(\cdot, \lambda), B_{\gamma}\left(H^{1}(\Omega)\right), 0\right)=1$.

Remark 3.2. Consider $\mathbb{V}=\mathbb{R}^{n}$ as an orthogonal $S O(2)$-representation and let $\Omega \subset \mathbb{V}$ be $S O(2)$-invariant. Then $H^{1}(\Omega)$ is an orthogonal $S O(2)$-representation with an action given by $(g u)(x)=u(g x)$. For every $\lambda_{i} \in \sigma(-\Delta ; \Omega), \mathbb{V}_{-\Delta}\left(\lambda_{i}\right)$ is an orthogonal finite-dimensional $S O(2)$-representation. Moreover, since $\Psi$ is $S O(2)$-invariant, $\nabla_{u} \Psi$ is $S O(2)$-equivariant.

Lemma 3.2. Assume that $\Omega \subset \mathbb{V}$ is $S O(2)$-invariant. Fix $\lambda \notin \sigma(-\Delta ; \Omega)$ and $\gamma>0$. Then

$$
\nabla_{S O(2)}-\operatorname{deg}\left(\nabla_{u} \Psi(\cdot, \lambda), B_{\gamma}\left(H^{1}(\Omega)\right)\right)=\prod_{\lambda_{i}<\lambda} \nabla_{S O(2)}-\operatorname{deg}\left(-I d, B_{\gamma}\left(\mathbb{V}_{-\Delta}\left(\lambda_{i}\right)\right)\right) \in U(S O(2)) .
$$

It is understood that if $\lambda<0$, then

$$
\nabla_{S O(2)}-\operatorname{deg}\left(\nabla_{u} \Psi(\cdot, \lambda), B_{\gamma}\left(H^{1}(\Omega)\right)\right)=\mathbb{I} \in U(S O(2)) .
$$


Proof. ¿From (3.3) it follows that $\sigma((1+\lambda) \mathcal{K})=\left\{\frac{\lambda+1}{\lambda_{i}+1}: \lambda_{i} \in \sigma(-\Delta ; \Omega)\right\}$. By assumption $1 \notin \sigma((1+\lambda) \mathcal{K})$. Applying Theorem 2.4 we obtain

$$
\begin{gathered}
\nabla_{S O(2)}-\operatorname{deg}\left(\nabla \Psi_{u}(\cdot, \lambda), B_{\gamma}\left(H^{1}(\Omega)\right)\right)=\nabla_{S O(2)}-\operatorname{deg}\left(I d-(\lambda+1) \mathcal{K}, B_{\gamma}\left(H^{1}(\Omega)\right)\right)= \\
=\prod_{\frac{\lambda+1}{\lambda_{i}+1}>1} \nabla_{S O(2)}-\operatorname{deg}\left(-I d, B_{\gamma}\left(\mathbb{V}_{-\Delta}\left(\lambda_{i}\right)\right)\right),
\end{gathered}
$$

which completes the proof.

\section{Results}

In this section we formulate and prove the main results of this article.

In the first subsection we formulate the sufficient conditions for the existence of nonconstant solutions of the following equation

$$
\left\{\begin{aligned}
-\Delta u & =f(u) & & \text { in } \Omega, \\
\frac{\partial u}{\partial \nu} & =0 & & \text { on } \partial \Omega .
\end{aligned}\right.
$$

In the second subsection we study continuation of solutions of the following family of equations

$$
\left\{\begin{aligned}
-\Delta u & =f(u, \lambda) & & \text { in } \Omega, \\
\frac{\partial u}{\partial \nu} & =0 & & \text { on } \partial \Omega,
\end{aligned}\right.
$$

Finally in the third subsection we study global bifurcations from infinity of solutions of above problem.

In the proofs of theorems of this section as the topological invariants we use the LeraySchauder degree and the degree for $\mathrm{SO}(2)$-equivariant gradient maps.

4.1. Existence of nonconstant solutions. In this section we study weak solutions of the following equation

$$
\left\{\begin{aligned}
-\Delta u & =f(u) & & \text { in } \Omega, \\
\frac{\partial u}{\partial \nu} & =0 & & \text { on } \partial \Omega,
\end{aligned}\right.
$$

where $\Omega \subset \mathbb{R}^{n}$ is an open, bounded set with $C^{1-}$-boundary and $f \in C^{1}(\mathbb{R}, \mathbb{R})$ satisfy the following assumption

(A.1) $\left|f^{\prime}(x)\right| \leq a+b|x|^{p}$ for some $a, b>0$, where $1<p<\frac{4}{n-2}$, for $n \geq 3$ and $1<p<\infty$ for $n=1,2$.

Set $F: \mathbb{R} \rightarrow \mathbb{R}$ a primitive of $f$ i.e. $F(t)=\int_{0}^{t} f(s) d s$. Weak solutions of equation (4.1.1) are in one to one correspondence with critical points of a functional $\Phi \in C^{2}\left(H^{1}(\Omega), \mathbb{R}\right)$ defined by $\Phi(u)=\frac{1}{2} \int_{\Omega}|\nabla u|^{2} d x-\int_{\Omega} F(u) d x$. 
Remark 4.1.1. Constant function $z_{0} \in H^{1}(\Omega)$ is a critical point of $\Phi$ iff $z_{0} \in Z=f^{-1}(0)$. Fix $z_{0} \in Z$. Since $\nabla^{2} \Phi\left(z_{0}\right)=I d-\left(1+f^{\prime}\left(z_{0}\right)\right) \mathcal{K}$ and (3.3) it follows that $\nabla^{2} \Phi\left(z_{0}\right): H^{1}(\Omega) \rightarrow$ $H^{1}(\Omega)$ is an isomorphism iff $f^{\prime}\left(z_{0}\right) \notin \sigma(-\Delta ; \Omega)$.

Let us put the following additional assumption

(A.2) there exists limit $f^{\prime}(\infty)=\lim _{|x| \rightarrow \infty} \frac{f(x)}{x}$.

Notice that $\nabla \Phi(u)=\nabla^{2} \Phi(\infty) u+o\left(|u|_{H^{1}(\Omega)}\right)=u-\left(1+f^{\prime}(\infty)\right) \mathcal{K} u+o\left(|u|_{H^{1}(\Omega)}\right)$ as $|u|_{H^{1}(\Omega)} \rightarrow \infty$.

We treat $\infty$ as a critical point of $\Phi$. We say that $\infty$ is an isolated critical point of $\Phi$ if $(\nabla \Phi)^{-1}(0)$ is bounded. Assume that all the elements of $Z \cup\{\infty\}$ are isolated critical points of $\Phi$. From now on $\gamma_{z}$ denotes a positive real number such that:

(i) if $z \in Z$, then $(\nabla \Phi)^{-1}(0) \cap D_{\gamma_{z}}\left(H^{1}(\Omega), z\right)=\{z\}$,

(ii) if $z=\infty$, then $(\nabla \Phi)^{-1}(0) \subset B_{\gamma_{\infty}}\left(H^{1}(\Omega)\right)$.

Lemma 4.1.1. Assume that assumption (A.1) is fulfilled, $z_{0} \in Z$ and $f^{\prime}\left(z_{0}\right) \notin \sigma(-\Delta ; \Omega)$. Then $\operatorname{deg}_{\mathrm{LS}}\left(\nabla \Phi, B_{\gamma_{z_{0}}}\left(H^{1}(\Omega), z_{0}\right), 0\right)=(-1)^{\nu\left(f^{\prime}\left(z_{0}\right)\right)}$.

Proof. It is easy to see that $\nabla^{2} \Phi\left(z_{0}\right)=I d-\left(1+f^{\prime}\left(z_{0}\right)\right) \mathcal{K}$. Since $f^{\prime}\left(z_{0}\right) \notin \sigma(-\Delta ; \Omega)$, $\nabla^{2} \Phi\left(z_{0}\right)$ is an isomorphism. From the properties of the Leray-Schauder degree we get $\left.\operatorname{deg}_{\mathrm{LS}}\left(\nabla \Phi, B_{\gamma_{z_{0}}}\left(H^{1}(\Omega), z_{0}\right), 0\right)=\operatorname{deg}_{\mathrm{LS}}\left(\nabla^{2} \Phi\left(z_{0}\right), B_{\gamma_{z_{0}}}\left(H^{1}(\Omega)\right), 0\right)\right)$. The rest of the prove is a direct consequence of Lemma 3.1 .

Since the proof of the next lemma is similar to the proof of Lemma 4.1.1, we will omit it.

Lemma 4.1.2. Assume that assumptions (A.1), (A.2) are satisfied and that $f^{\prime}(\infty) \notin$ $\sigma(-\Delta ; \Omega)$. Then $\operatorname{deg}_{\mathrm{LS}}\left(\nabla \Phi, B_{\gamma_{\infty}}\left(H^{1}(\Omega)\right), 0\right)=(-1)^{\nu\left(f^{\prime}(\infty)\right)}$.

Put the following assumptions:

(A.3) $\# Z<\infty$,

(A.4) $f^{\prime}(z) \notin \sigma(-\Delta ; \Omega)$ for every $z \in Z \cup\{\infty\}$.

Define $Z_{+}:=\left\{z \in Z: f^{\prime}(z)>0\right\}, Z_{-}:=\left\{z \in Z: f^{\prime}(z)<0\right\}$.

Notice that if assumption (A.4) is fulfilled, then $Z_{+} \cup Z_{-}=Z$.

In the next theorem we ensure the existence of nonconstant solutions of equation (4.1.1).

Theorem 4.1.1. Suppose that assumptions (A.1)-(A.4) are fulfilled. Moreover, assume that

(1) if $f^{\prime}(\infty)<0$, then there exists $z_{0} \in Z_{+}$such that $\nu\left(f^{\prime}\left(z_{0}\right)\right)$ is even,

(2) if $f^{\prime}(\infty)>0$ and $\nu\left(f^{\prime}(\infty)\right)$ is odd, then there exists $z_{0} \in Z_{+}$such that $\nu\left(f^{\prime}\left(z_{0}\right)\right)$ is even,

(3) if $f^{\prime}(\infty)>0$ and $\nu\left(f^{\prime}(\infty)\right)$ is even, then $\#\left\{z \in Z_{+}: \nu\left(f^{\prime}(z)\right)\right.$ is even $\} \neq 1$.

Then there exists at least one nonconstant solution of equation (4.1.1).

Proof. By the properties of the Leray-Schauder degree we obtain that

$$
\operatorname{deg}_{\mathrm{LS}}\left(\nabla \Phi, B_{\gamma_{\infty}}\left(H^{1}(\Omega)\right) \backslash \bigcup_{z \in Z} D_{\gamma_{z}}\left(H^{1}(\Omega), z\right), 0\right)=
$$




$$
=\operatorname{deg}_{\mathrm{LS}}\left(\nabla \Phi, B_{\gamma_{\infty}}\left(H^{1}(\Omega)\right), 0\right)-\sum_{z \in Z} \operatorname{deg}_{\mathrm{LS}}\left(\nabla \Phi, B_{\gamma_{z}}\left(H^{1}(\Omega), z\right), 0\right) .
$$

What is left is to show that

$$
\operatorname{deg}_{\mathrm{LS}}\left(\nabla \Phi, B_{\gamma_{\infty}}\left(H^{1}(\Omega)\right), 0\right) \neq \sum_{z \in Z} \operatorname{deg}_{\mathrm{LS}}\left(\nabla \Phi, B_{\gamma_{z}}\left(H^{1}(\Omega), z\right), 0\right) .
$$

Suppose, contrary to our claim, that

$$
\operatorname{deg}_{\mathrm{LS}}\left(\nabla \Phi, B_{\gamma_{\infty}}\left(H^{1}(\Omega)\right), 0\right)=\sum_{z \in Z} \operatorname{deg}_{\mathrm{LS}}\left(\nabla \Phi, B_{\gamma_{z}}\left(H^{1}(\Omega), z\right), 0\right) .
$$

By Lemma 4.1.1 we obtain

$$
\sum_{z \in Z_{-}} \operatorname{deg}_{\mathrm{LS}}\left(\nabla \Phi, B_{\gamma_{z}}\left(H^{1}(\Omega), z\right), 0\right)=\# Z_{-} .
$$

Now put $Z_{+}^{o}:=\left\{z \in Z_{+}: \nu\left(f^{\prime}(z)\right)\right.$ is odd $\}$ and $Z_{+}^{e}:=\left\{z \in Z_{+}: \nu\left(f^{\prime}(z)\right)\right.$ is even $\}$. Then $Z_{+}=Z_{+}^{o} \cup Z_{+}^{e}$ and $Z_{+}^{o} \cap Z_{+}^{e}=\emptyset$. Again from Lemma 4.1.1 it follows that

$$
\sum_{z \in Z_{+}} \operatorname{deg}_{\mathrm{LS}}\left(\nabla \Phi, B_{\gamma_{z}}\left(H^{1}(\Omega), z\right), 0\right)=\# Z_{+}^{e}-\# Z_{+}^{o}
$$

By Lemma 4.1.2 we have if $f^{\prime}(\infty)<0$, then $\operatorname{deg}_{L S}\left(\nabla \Phi, B_{\gamma_{\infty}}\left(H^{1}(\Omega)\right), 0\right)=1$. Moreover, if $f^{\prime}(\infty)>0$ and $\nu\left(f^{\prime}(\infty)\right)$ is odd, then $\operatorname{deg}_{\mathrm{LS}}\left(\nabla \Phi, B_{\gamma_{\infty}}\left(H^{1}(\Omega)\right), 0\right)=-1$.

Let assumption (1) or (2) be fulfilled. Then $\operatorname{deg}_{\mathrm{LS}}\left(\nabla \Phi, B_{\gamma_{\infty}}\left(H^{1}(\Omega)\right), 0\right)=-\operatorname{sign} f^{\prime}(\infty)$. From this and equations (4.1.2)-(4.1.4) we obtain $\# Z_{+}^{e}-\# Z_{+}^{o}+\# Z_{-}=-\operatorname{sign} f^{\prime}(\infty)$. Moreover, it is easy to see that $\# Z_{+}-\# Z_{-}=\operatorname{sign} f^{\prime}(\infty)$. Hence

$$
\left\{\begin{array}{llr}
\# Z_{+}^{e}-\# Z_{+}^{o}+\# Z_{-}= & -\operatorname{sign} f^{\prime}(\infty), \\
\# Z_{+}-\# Z_{-} & = & \operatorname{sign} f^{\prime}(\infty) .
\end{array}\right.
$$

We thus get $\# Z_{+}^{e}=0$, a contradiction.

(3) By Lemma 4.1.2 we obtain $\operatorname{deg}_{\mathrm{LS}}\left(\nabla \Phi, B_{\gamma_{\infty}}\left(H^{1}(\Omega)\right), 0\right)=1$. Therefore

$$
\begin{cases}\# Z_{+}^{e}-\# Z_{+}^{o}+\# Z_{-} & =1, \\ \# Z_{+}-\# Z_{-} & =1,\end{cases}
$$

which implies $\# Z_{+}^{e}=1$, a contradiction.

From now on we assume that

(A.5) $\mathbb{V}=\mathbb{R}^{n}$ is a nontrivial orthogonal $S O(2)$-representation and that $\Omega \subset \mathbb{V}$ is $S O(2)$ invariant.

Remark 4.1.2. Since $\Omega \subset \mathbb{V}$ is $S O(2)$-invariant, $H^{1}(\Omega)$ is an orthogonal $S O(2)$-representation, with $S O(2)$-action defined by $(g u)(x)=u(g x)$, and $\Phi \in C_{S O(2)}^{2}\left(H^{1}(\Omega), \mathbb{R}\right)$. Hence $\nabla \Phi \in C_{S O(2)}^{1}\left(H^{1}(\Omega), H^{1}(\Omega)\right)$.

The following two lemmas are similar to 4.1.1, 4.1.2, respectively. Since $\nabla \Phi$ is $S O(2)$ invariant, instead of the Leray-Schauder degree we will apply the degree for $S O(2)$-equivariant gradient maps. 
Lemma 4.1.3. Assume that assumptions (A.1), (A.5) are fulfilled. Fix $z_{0} \in Z$ such that $f^{\prime}\left(z_{0}\right) \notin \sigma(-\Delta ; \Omega)$. If $z_{0} \in Z_{+}$, then

$$
\begin{gathered}
\nabla_{S O(2)}-\operatorname{deg}\left(\nabla \Phi, B_{\gamma_{z_{0}}}\left(H^{1}(\Omega), z_{0}\right)\right)= \\
=\prod_{\lambda_{i}<f^{\prime}\left(z_{0}\right)} \nabla_{S O(2)}-\operatorname{deg}\left(-I d, B_{\gamma_{z_{0}}}\left(\mathbb{V}_{-\Delta}\left(\lambda_{i}\right)\right)\right) \in U(S O(2)) .
\end{gathered}
$$

Moreover, if $z_{0} \in Z_{-}$, then $\nabla_{S O(2)}-\operatorname{deg}\left(\nabla \Phi, B_{\gamma_{z_{0}}}\left(H^{1}(\Omega), z_{0}\right)\right)=\mathbb{I} \in U(S O(2))$.

Proof. Since $z_{0} \in H^{1}(\Omega)$ is a constant function, $B_{\gamma_{z_{0}}}\left(H^{1}(\Omega), z_{0}\right) \subset H^{1}(\Omega)$ is $S O(2)$-invariant. Moreover, $\nabla \Phi$ is an $S O(2)$-equivariant operator of the form compact perturbation of the identity. Hence $\nabla_{S O(2)}-\operatorname{deg}\left(\nabla \Phi, B_{\gamma}\left(H^{1}(\Omega), z_{0}\right)\right) \in U(S O(2))$ is well-defined. It is clear that $\nabla^{2} \Phi\left(z_{0}\right)=I d-\left(f^{\prime}\left(z_{0}\right)+1\right) \mathcal{K}$ and that $\nabla^{2} \Phi\left(z_{0}\right)$ is an isomorphism. From Theorem 2.1 we have

$$
\nabla_{S O(2)}-\operatorname{deg}\left(\nabla \Phi, B_{\gamma_{z_{0}}}\left(H^{1}(\Omega), z_{0}\right)\right)=\nabla_{S O(2)}-\operatorname{deg}\left(\nabla^{2} \Phi\left(z_{0}\right), B_{\gamma_{z_{0}}}\left(H^{1}(\Omega)\right)\right) .
$$

The rest of the proof is a direct consequence of Lemma 3.2

Lemma 4.1.4. Assume that assumptions (A.1), (A.2), (A.5) are satisfied and that $f^{\prime}(\infty) \notin \sigma(-\Delta ; \Omega)$. Then

(1) if $f^{\prime}(\infty)>0$, then

$$
\begin{gathered}
\nabla_{S O(2)}-\operatorname{deg}\left(\nabla \Phi, B_{\gamma_{\infty}}\left(H^{1}(\Omega)\right)\right)= \\
\prod_{\lambda_{i}<f^{\prime}(\infty)} \nabla_{S O(2)}-\operatorname{deg}\left(-I d, B_{\gamma_{\infty}}\left(\mathbb{V}_{-\Delta}\left(\lambda_{i}\right)\right)\right) \in U(S O(2)),
\end{gathered}
$$

(2) if $f^{\prime}(\infty)<0$, then $\nabla_{S O(2)}-\operatorname{deg}\left(\nabla \Phi, B_{\gamma_{\infty}}\left(H^{1}(\Omega)\right)\right)=\mathbb{I} \in U(S O(2))$.

Proof. Since $\nabla \Phi$ is an $S O(2)$-equivariant operator of the form compact perturbation of the identity and $\nabla^{2} \Phi(\infty)=I d-\left(1+f^{\prime}(\infty)\right) \mathcal{K}$ is an isomorphism,

$$
\nabla_{S O(2)}-\operatorname{deg}\left(\nabla \Phi, B_{\gamma_{\infty}}\left(H^{1}(\Omega)\right)\right)=\nabla_{S O(2)}-\operatorname{deg}\left(\nabla^{2} \Phi(\infty), B_{\gamma_{\infty}}\left(H^{1}(\Omega)\right)\right) .
$$

The rest of the proof is a direct consequence of Lemma 3.2

The following corollary is an immediate consequence of Lemmas [2.1, 4.1.3, 4.1.4,

Corollary 4.1.1. If $z \in Z$ and assumptions of Lemma 4.1.3 are satisfied, then

(1) if $H \in \Upsilon(S O(2))$ and $\nabla_{S O(2)}-\operatorname{deg}_{H}\left(\nabla \Phi, B_{\gamma_{z}}\left(H^{1}(\Omega), z\right)\right) \neq 0$, then

$$
\operatorname{sign}\left(\nabla_{S O(2)}-\operatorname{deg}_{H}\left(\nabla \Phi, B_{\gamma_{z}}\left(H^{1}(\Omega), z\right)\right)\right)=(-1)^{\nu\left(f^{\prime}(z)\right)},
$$

(2) $\nabla_{S O(2)}-\operatorname{deg}_{S O(2)}\left(\nabla \Phi, B_{\gamma_{z}}\left(H^{1}(\Omega), z\right)\right)=(-1)^{\nu\left(f^{\prime}(z)\right)}$.

If $z=\infty$ and assumptions of Lemma 4.1.4 are fulfilled, then

(1) if $H \in \Upsilon(S O(2))$ and $\nabla_{S O(2)}-\operatorname{deg}_{H}\left(\nabla \Phi, B_{\gamma_{\infty}}\left(H^{1}(\Omega)\right)\right) \neq 0$, then

$$
\operatorname{sign}\left(\nabla_{S O(2)}-\operatorname{deg}_{H}\left(\nabla \Phi, B_{\gamma_{\infty}}\left(H^{1}(\Omega)\right)\right)\right)=(-1)^{\nu\left(f^{\prime}(\infty)\right)},
$$

(2) $\nabla_{S O(2)}-\operatorname{deg}_{S O(2)}\left(\nabla \Phi, B_{\gamma_{\infty}}\left(H^{1}(\Omega)\right)\right)=(-1)^{\nu\left(f^{\prime}(\infty)\right)}$. 
Define $\lambda_{0}=\min \left\{\lambda_{i} \in \sigma(-\Delta ; \Omega): \mathbb{V}_{-\Delta}\left(\lambda_{i}\right)\right.$ is a nontrivial $S O(2)$-representation $\}$. Moreover, for $z \in Z \cup\{\infty\}$ define $\mathbb{V}\left(f^{\prime}(z)\right)=\bigoplus_{\lambda_{i}<f^{\prime}(z)} \mathbb{V}_{-\Delta}\left(\lambda_{i}\right)$.

In the next three theorems we prove the existence of nonconstant solutions of equation (4.1.1). Since $\Omega \subset \mathbb{V}$ is $S O(2)$-invariant, $\nabla \Phi$ is $S O(2)$-equivariant. Therefore we use in the proofs the degree for $S O(2)$-equivariant gradient maps.

It is worth to point out that we obtain the existence of nonconstant solutions of equation (4.1.1) also if the assumptions of Theorem 4.1.1 are not fulfilled.

Theorem 4.1.2. Suppose that assumptions (A.1)-(A.5) are fulfilled. Moreover, assume that $f^{\prime}(\infty)<0$ and that there exists $z_{0} \in Z_{+}$such that $\lambda_{0}<f^{\prime}\left(z_{0}\right)$. Then there exists at least one nonconstant solution of equation (4.1.1).

Proof. In view of Theorem 4.1.1, to complete the proof, it is enough to assume that $\nu\left(f^{\prime}(z)\right)$ is odd for all $z \in Z_{+}$. By the properties of the degree for $S O(2)$-equivariant gradient maps we obtain

$$
\begin{gathered}
\nabla_{S O(2)}-\operatorname{deg}\left(\nabla \Phi, B_{\gamma_{\infty}}\left(H^{1}(\Omega)\right) \backslash \bigcup_{z \in Z} D_{\gamma_{z}}\left(H^{1}(\Omega), z\right)\right)= \\
=\nabla_{S O(2)}-\operatorname{deg}\left(\nabla \Phi, B_{\gamma_{\infty}}\left(H^{1}(\Omega)\right)\right)-\sum_{z \in Z} \nabla_{S O(2)}-\operatorname{deg}\left(\nabla \Phi, B_{\gamma_{z}}\left(H^{1}(\Omega), z\right)\right) .
\end{gathered}
$$

Therefore, to complete the proof, it remains to prove that

$$
\nabla_{S O(2)}-\operatorname{deg}\left(\nabla \Phi, B_{\gamma_{\infty}}\left(H^{1}(\Omega)\right)\right) \neq \sum_{z \in Z} \nabla_{S O(2)}-\operatorname{deg}\left(\nabla \Phi, B_{\gamma_{z}}\left(H^{1}(\Omega), z\right)\right) .
$$

Suppose, contrary to our claim, that

$$
\nabla_{S O(2)}-\operatorname{deg}\left(\nabla \Phi, B_{\gamma_{\infty}}\left(H^{1}(\Omega)\right)\right)=\sum_{z \in Z} \nabla_{S O(2)}-\operatorname{deg}\left(\nabla \Phi, B_{\gamma_{z}}\left(H^{1}(\Omega), z\right)\right) .
$$

Since $\mathbb{V}_{-\Delta}\left(\lambda_{0}\right)$ is a nontrivial $S O(2)$-representation, there is $k^{\prime} \in \mathbb{N}$ such that $\mathbb{V}_{-\Delta}\left(\lambda_{0}\right)=$ $=\mathbb{R}\left[1, k^{\prime}\right] \oplus \mathbb{R}\left[1, k^{\prime}\right]^{\perp}$. From (4.1.5) we get

$$
\nabla_{S O(2)}-\operatorname{deg}_{\mathbb{Z}_{k^{\prime}}}\left(\nabla \Phi, B_{\gamma_{\infty}}\left(H^{1}(\Omega)\right)\right)=\sum_{z \in Z} \nabla_{S O(2)}-\operatorname{deg}_{\mathbb{Z}_{k^{\prime}}}\left(\nabla \Phi, B_{\gamma_{z}}\left(H^{1}(\Omega), z\right)\right) .
$$

Since $f^{\prime}(\infty)<0$ and Lemma 4.1.4 we obtain

$$
\nabla_{S O(2)}-\operatorname{deg}_{\mathbb{Z}_{k^{\prime}}}\left(\nabla \Phi, B_{\gamma_{\infty}}\left(H^{1}(\Omega)\right)\right)=0
$$

If $z \in Z_{-}$, then, by Lemma 4.1.3, we have

$$
\nabla_{S O(2)}-\operatorname{deg}_{\mathbb{Z}_{k^{\prime}}}\left(\nabla \Phi, B_{\gamma_{z}}\left(H^{1}(\Omega), z\right)\right)=0 .
$$

Taking into account (4.1.6), (4.1.7) and (4.1.8) we obtain

$$
\sum_{z \in Z_{+}} \nabla_{S O(2)}-\operatorname{deg}_{\mathbb{Z}_{k^{\prime}}}\left(\nabla \Phi, B_{\gamma_{z}}\left(H^{1}(\Omega), z\right)\right)=0 .
$$


Fix $z \in Z_{+}$. From Lemma 4.1.3 we have

$$
\begin{gathered}
\nabla_{S O(2)}-\operatorname{deg}\left(\nabla \Phi, B_{\gamma_{z}}\left(H^{1}(\Omega), z\right)\right)=\prod_{\lambda_{i}<f^{\prime}(z)} \nabla_{S O(2)}-\operatorname{deg}\left(-I d, B_{\gamma_{z}}\left(\mathbb{V}_{-\Delta}\left(\lambda_{i}\right)\right)\right)= \\
=\nabla_{S O(2)}-\operatorname{deg}\left(-I d, B_{\gamma_{z}}\left(\mathbb{V}\left(f^{\prime}(z)\right)\right) .\right.
\end{gathered}
$$

By assumption $\nu\left(f^{\prime}(z)\right)$ is odd. Hence from Corollary 4.1.1 we obtain

$\nabla_{S O(2)}-\operatorname{deg}_{S O(2)}\left(\nabla \Phi, B_{\gamma_{z}}\left(H^{1}(\Omega), z\right)\right)=-1$ and $\nabla_{S O(2)}-\operatorname{deg}_{\mathbb{Z}_{k^{\prime}}}\left(\nabla \Phi, B_{\gamma_{z}}\left(H^{1}(\Omega), z\right)\right) \leq 0$. Using the above and (4.1.9) we get $\nabla_{S O(2)}-\operatorname{deg}_{\mathbb{Z}_{k^{\prime}}}\left(\nabla \Phi, B_{\gamma}\left(H^{1}(\Omega), z\right)\right)=0$ for all $z \in Z_{+}$. By the assumption there exists $z_{0} \in Z_{+}$such that $f^{\prime}\left(z_{0}\right)>\lambda_{0}$. Therefore $\mathbb{V}\left(f^{\prime}\left(z_{0}\right)\right)=$ $\mathbb{R}\left[1, k^{\prime}\right] \oplus \mathbb{R}\left[1, k^{\prime}\right]^{\perp}$. Finally, by Lemmas 2.1 , 4.1.3, we obtain

$$
\nabla_{S O(2)}-\operatorname{deg}_{\mathbb{Z}_{k^{\prime}}}\left(\nabla \Phi, B_{\gamma_{z_{0}}}\left(H^{1}(\Omega), z_{0}\right)\right)=\nabla_{S O(2)}-\operatorname{deg}_{\mathbb{Z}_{k^{\prime}}}\left(-I d, B_{\gamma_{z_{0}}}\left(\mathbb{V}\left(f^{\prime}\left(z_{0}\right)\right)\right)\right) \neq 0
$$

a contradiction.

Theorem 4.1.3. Suppose that assumptions (A.1)-(A.5) are fulfilled, $f^{\prime}(\infty)>0$ and $\nu\left(f^{\prime}(\infty)\right)$ is odd. Additionally, assume that one of the following conditions is satisfied

(1) there are $z_{0}, z_{1} \in Z_{+}$such that $f^{\prime}\left(z_{0}\right) \geq f^{\prime}\left(z_{1}\right)>\lambda_{0}$ and $f^{\prime}\left(z_{0}\right)>f^{\prime}(\infty)$,

(2) there exists exactly one $z_{0} \in Z_{+}$such that

(a) $f^{\prime}\left(z_{0}\right)>\lambda_{0}$,

(b) there exists $\lambda_{i_{0}} \in \sigma(-\Delta ; \Omega)$ such that $f^{\prime}\left(z_{0}\right)<\lambda_{i_{0}}<f^{\prime}(\infty)\left(\right.$ or $f^{\prime}(\infty)<\lambda_{i_{0}}<$ $\left.f^{\prime}\left(z_{0}\right)\right)$ and that $\mathbb{V}_{-\Delta}\left(\lambda_{i_{0}}\right)$ is a nontrivial $S O(2)$-representation,

(3) there exists $\lambda_{i_{0}} \in \sigma(-\Delta ; \Omega)$ such that

(a) $f^{\prime}(z)<\lambda_{i_{0}}<f^{\prime}(\infty)$ for all $z \in Z_{+}$,

(b) there exists $k^{\prime} \in \mathbb{N}$ such that

(i) $\mathbb{V}_{-\Delta}\left(\lambda_{i_{0}}\right)=\mathbb{R}\left[1, k^{\prime}\right] \oplus \mathbb{R}\left[1, k^{\prime}\right]^{\perp}$,

(ii) $\mathbb{R}\left[1, k^{\prime}\right] \not \subset \mathbb{V}_{-\Delta}\left(\lambda_{i}\right)$ for $\lambda_{i} \in \sigma(-\Delta ; \Omega) \cap\left(-\infty, \lambda_{i_{0}}\right)$.

Then there exists at least one nonconstant solution of equation (4.1.1).

Proof. The proof is similar to that of Theorem 4.1.2. By the properties of the degree for $S O(2)$-equivariant gradient maps we obtain

$$
\begin{gathered}
\nabla_{S O(2)}-\operatorname{deg}\left(\nabla \Phi, B_{\gamma_{\infty}}\left(H^{1}(\Omega)\right) \backslash \bigcup_{z \in Z} D_{\gamma_{z}}\left(H^{1}(\Omega), z\right)\right)= \\
=\nabla_{S O(2)}-\operatorname{deg}\left(\nabla \Phi, B_{\gamma_{\infty}}\left(H^{1}(\Omega)\right)\right)-\sum_{z \in Z} \nabla_{S O(2)}-\operatorname{deg}\left(\nabla \Phi, B_{\gamma_{z}}\left(H^{1}(\Omega), z\right)\right) .
\end{gathered}
$$

It remains to prove that

$$
\nabla_{S O(2)}-\operatorname{deg}\left(\nabla \Phi, B_{\gamma_{\infty}}\left(H^{1}(\Omega)\right)\right) \neq \sum_{z \in Z} \nabla_{S O(2)}-\operatorname{deg}\left(\nabla \Phi, B_{\gamma_{z}}\left(H^{1}(\Omega), z\right)\right) .
$$

Suppose, contrary to our claim, that

$$
\nabla_{S O(2)}-\operatorname{deg}\left(\nabla \Phi, B_{\gamma_{\infty}}\left(H^{1}(\Omega)\right)\right)=\sum_{z \in Z} \nabla_{S O(2)}-\operatorname{deg}\left(\nabla \Phi, B_{\gamma_{z}}\left(H^{1}(\Omega), z\right)\right) .
$$


If $z \in Z_{-}$and $k \in \mathbb{N}$, then, by Lemma4.1.3, we get $\nabla_{S O(2)}-\operatorname{deg}_{\mathbb{Z}_{k}}\left(\nabla \Phi, B_{\gamma_{z}}\left(H^{1}(\Omega), z\right)\right)=0$ and

$$
\nabla_{S O(2)}-\operatorname{deg}_{\mathbb{Z}_{k}}\left(\nabla \Phi, B_{\gamma_{\infty}}\left(H^{1}(\Omega)\right)\right)=\sum_{z \in Z_{+}} \nabla_{S O(2)}-\operatorname{deg}_{\mathbb{Z}_{k}}\left(\nabla \Phi, B_{\gamma_{z}}\left(H^{1}(\Omega), z\right)\right) .
$$

From Theorem 4.1.1 it follows that, to complete the proof, it suffices to consider the case $\nu\left(f^{\prime}(z)\right)$ is odd for all $z \in Z_{+} \cup\{\infty\}$. Therefore, by Corollary 4.1.1 we obtain that $\nabla_{S O(2)}-\operatorname{deg}_{\mathbb{Z}_{k}}\left(\nabla \Phi, B_{\gamma_{z}}\left(H^{1}(\Omega), z\right)\right) \leq 0$ for all $z \in Z_{+} \cup\{\infty\}$ and $k \in \mathbb{N}$.

(1) Since $\mathbb{V}_{-\Delta}\left(\lambda_{0}\right)$ is a nontrivial $S O(2)$-representation there is $k^{\prime} \in \mathbb{N}$ such that $\mathbb{V}_{-\Delta}\left(\lambda_{0}\right)=$ $\mathbb{R}\left[1, k^{\prime}\right] \oplus \mathbb{R}\left[1, k^{\prime}\right]^{\perp}$. Hence, by Lemma 2.1] we have

$$
\nabla_{S O(2)}-\operatorname{deg}_{\mathbb{Z}_{k^{\prime}}}\left(\nabla \Phi, B_{\gamma_{z_{0}}}\left(H^{1}(\Omega), z_{0}\right)\right), \nabla_{S O(2)}-\operatorname{deg}_{\mathbb{Z}_{k^{\prime}}}\left(\nabla \Phi, B_{\gamma_{z_{1}}}\left(H^{1}(\Omega), z_{1}\right)\right)<0 .
$$

Since $f^{\prime}\left(z_{0}\right)>f^{\prime}(\infty)$, it follows that $\mathbb{V}\left(f^{\prime}(\infty)\right) \subset \mathbb{V}\left(f^{\prime}\left(z_{0}\right)\right)$ and consequently, by Lemmas 2.1, 4.1.3, 4.1.4, we obtain

$$
\begin{aligned}
& \nabla_{S O(2)}-\operatorname{deg}_{\mathbb{Z}_{k^{\prime}}}(\nabla \Phi\left., B_{\gamma_{z_{0}}}\left(H^{1}(\Omega), z_{0}\right)\right)=\nabla_{S O(2)}-\operatorname{deg}_{\mathbb{Z}_{k^{\prime}}}\left(\nabla^{2} \Phi\left(z_{0}\right), B_{\gamma_{z_{0}}}\left(H^{1}(\Omega), z_{0}\right)\right)= \\
&=\nabla_{S O(2)}-\operatorname{deg}_{\mathbb{Z}_{k^{\prime}}}\left(-I d, B_{\gamma_{z_{0}}}\left(\mathbb{V}\left(f^{\prime}\left(z_{0}\right)\right)\right) \leq\right. \\
& \leq \nabla_{S O(2)}-\operatorname{deg}_{\mathbb{Z}_{k^{\prime}}}\left(-I d, B_{\gamma_{\infty}}\left(\mathbb{V}\left(f^{\prime}(\infty)\right)\right)\right)= \\
&=\nabla_{S O(2)}-\operatorname{deg}_{\mathbb{Z}_{k^{\prime}}}\left(\nabla^{2} \Phi(\infty), B_{\gamma_{\infty}}\left(H^{1}(\Omega)\right)\right)=\nabla_{S O(2)}-\operatorname{deg}_{\mathbb{Z}_{k^{\prime}}}\left(\nabla \Phi, B_{\gamma_{\infty}}\left(H^{1}(\Omega)\right)\right) .
\end{aligned}
$$

Taking together the above inequalities and (4.1.10) we obtain

$$
\begin{aligned}
& \nabla_{S O(2)}-\operatorname{deg}_{\mathbb{Z}_{k^{\prime}}}\left(\nabla \Phi, B_{\gamma_{\infty}}\left(H^{1}(\Omega)\right)\right) \geq \nabla_{S O(2)}-\operatorname{deg}_{\mathbb{Z}_{k^{\prime}}}\left(\nabla \Phi, B_{\gamma_{z_{0}}}\left(H^{1}(\Omega), z_{0}\right)\right)> \\
> & \nabla_{S O(2)}-\operatorname{deg}_{\mathbb{Z}_{k^{\prime}}}\left(\nabla \Phi, B_{\gamma_{z_{1}}}\left(H^{1}(\Omega), z_{1}\right)\right)+\nabla_{S O(2)}-\operatorname{deg}_{\mathbb{Z}_{k^{\prime}}}\left(\nabla \Phi, B_{\gamma_{z_{0}}}\left(H^{1}(\Omega), z_{0}\right) \geq\right. \\
\geq & \sum_{z \in Z_{+}} \nabla_{S O(2)}-\operatorname{deg}_{\mathbb{Z}_{k^{\prime}}}\left(\nabla \Phi, B_{\gamma_{z}}\left(H^{1}(\Omega), z\right)\right)=\nabla_{S O(2)}-\operatorname{deg}_{\mathbb{Z}_{k^{\prime}}}\left(\nabla \Phi, B_{\gamma_{\infty}}\left(H^{1}(\Omega)\right)\right),
\end{aligned}
$$

a contradiction.

(2) Since $\mathbb{V}_{-\Delta}\left(\lambda_{i_{0}}\right)$ is a nontrivial $S O(2)$-representation, there is $k^{\prime} \in \mathbb{N}$ such that $\mathbb{V}_{-\Delta}\left(\lambda_{i_{0}}\right)=\mathbb{R}\left[1, k^{\prime}\right] \oplus \mathbb{R}\left[1, k^{\prime}\right]^{\perp}$. Fix $z \in Z \backslash\left\{z_{0}\right\}$. Since $f^{\prime}(z)<\lambda_{0}, \mathbb{V}\left(f^{\prime}(z)\right)$ is a trivial $S O(2)$-representation, applying Lemmas 2.1, 4.1.3, we obtain

$$
\nabla_{S O(2)}-\operatorname{deg}_{\mathbb{Z}_{k^{\prime}}}\left(\nabla \Phi, B_{\gamma_{z}}\left(H^{1}(\Omega), z\right)\right)=0 .
$$

Thus

$$
\begin{gathered}
\nabla_{S O(2)}-\operatorname{deg}_{\mathbb{Z}_{k^{\prime}}}\left(\nabla \Phi, B_{\gamma_{\infty}}\left(H^{1}(\Omega)\right)=\sum_{z \in Z} \nabla_{S O(2)}-\operatorname{deg}_{\mathbb{Z}_{k^{\prime}}}\left(\nabla \Phi, B_{\gamma_{z}}\left(H^{1}(\Omega), z\right)\right)=\right. \\
=\sum_{z \in Z_{+}} \nabla_{S O(2)}-\operatorname{deg}_{\mathbb{Z}_{k^{\prime}}}\left(\nabla \Phi, B_{\gamma_{z}}\left(H^{1}(\Omega), z\right)\right)=\nabla_{S O(2)}-\operatorname{deg}_{\mathbb{Z}_{k^{\prime}}}\left(\nabla \Phi, B_{\gamma_{z_{0}}}\left(H^{1}(\Omega), z_{0}\right)\right) .
\end{gathered}
$$

Let $j_{0}, j_{\infty} \in \mathbb{N}$ be the largest integers such that

$$
\mathbb{V}\left(f^{\prime}\left(z_{0}\right)\right)=\mathbb{R}\left[j_{0}, k^{\prime}\right] \oplus \mathbb{R}\left[j_{0}, k^{\prime}\right]^{\perp}, \text { and } \mathbb{V}\left(f^{\prime}(\infty)\right)=\mathbb{R}\left[j_{\infty}, k^{\prime}\right] \oplus \mathbb{R}\left[j_{\infty}, k^{\prime}\right]^{\perp} .
$$

Since $f^{\prime}\left(z_{0}\right)<\lambda_{i_{0}}<f^{\prime}(\infty)$, we obtain $j_{0}<j_{\infty}$. Finally, by Lemmas 2.1, 4.1.3, we obtain

$$
\nabla_{S O(2)}-\operatorname{deg}_{\mathbb{Z}_{k^{\prime}}}\left(\nabla \Phi, B_{\gamma_{\infty}}\left(H^{1}(\Omega)\right)\right) \neq \nabla_{S O(2)}-\operatorname{deg}_{\mathbb{Z}_{k^{\prime}}}\left(\nabla \Phi, B_{\gamma_{z_{0}}}\left(H^{1}(\Omega), z_{0}\right)\right),
$$

a contradiction. The same proof remains valid if $f^{\prime}(\infty)<\lambda_{i_{0}}<f^{\prime}\left(z_{0}\right)$. 
(3) Since $\mathbb{R}\left[1, k^{\prime}\right] \not \subset \mathbb{V}\left(f^{\prime}(z)\right)$ for every $z \in Z_{+}$and Lemmas 2.1, 4.1.3,

$$
\nabla_{S O(2)}-\operatorname{deg}_{\mathbb{Z}_{k^{\prime}}}\left(\nabla \Phi, B_{\gamma_{z}}\left(H^{1}(\Omega), z\right)\right)=\nabla_{S O(2)}-\operatorname{deg}_{\mathbb{Z}_{k^{\prime}}}\left(-I d, B_{\gamma_{z}}\left(\mathbb{V}\left(f^{\prime}(z)\right)\right)=0,\right.
$$

for every $z \in Z$.

Thus, by the above and (4.1.10), we obtain

$$
\nabla_{S O(2)}-\operatorname{deg}_{\mathbb{Z}_{k^{\prime}}}\left(\nabla \Phi, B_{\gamma_{\infty}}\left(H^{1}(\Omega)\right)\right)=\sum_{z \in Z_{+}} \nabla_{S O(2)}-\operatorname{deg}_{\mathbb{Z}_{k^{\prime}}}\left(\nabla \Phi, B_{\gamma_{z}}\left(H^{1}(\Omega), z\right)\right)=0 .
$$

Since $\mathbb{V}_{-\Delta}\left(\lambda_{i_{0}}\right) \subset \mathbb{V}\left(f^{\prime}(\infty)\right), \mathbb{R}\left[1, k^{\prime}\right] \subset \mathbb{V}_{\infty}$ and consequently

$$
\nabla_{S O(2)}-\operatorname{deg}_{\mathbb{Z}_{k^{\prime}}}\left(\nabla \Phi, B_{\gamma_{\infty}}\left(H^{1}(\Omega)\right)\right)=\nabla_{S O(2)}-\operatorname{deg}_{\mathbb{Z}_{k^{\prime}}}\left(-I d, B_{\gamma_{\infty}}\left(\mathbb{V}\left(f^{\prime}(\infty)\right)\right) \neq 0,\right.
$$

a contradiction.

Theorem 4.1.4. Suppose that assumptions (A.1)-(A.5) are fulfilled, $f^{\prime}(\infty)>0$ and $\nu\left(f^{\prime}(\infty)\right)$ is even. Additionally assume that there exists $z_{0} \in Z_{+}$such that $\nu\left(f^{\prime}\left(z_{0}\right)\right)$ is even and one of the following conditions is fulfilled

(1) there exist $z_{1}, z_{2} \in\left(Z_{+} \cup\{\infty\}\right) \backslash\left\{z_{0}\right\}$ such that $f^{\prime}\left(z_{1}\right) \geq f^{\prime}\left(z_{2}\right)>\lambda_{0}$ and $f^{\prime}\left(z_{1}\right)>$ $f^{\prime}\left(z_{0}\right)$,

(2) there exists exactly one $z_{1} \in\left(Z_{+} \cup\{\infty\}\right) \backslash\left\{z_{0}\right\}$ such that

(a) $f^{\prime}\left(z_{1}\right)>\lambda_{0}$,

(b) there exists $\lambda_{i_{0}} \in \sigma(-\Delta ; \Omega)$ such that $f^{\prime}\left(z_{1}\right)<\lambda_{i_{0}}<f^{\prime}\left(z_{0}\right)$ (or $f^{\prime}\left(z_{0}\right)<\lambda_{i_{0}}<$ $\left.f^{\prime}\left(z_{1}\right)\right)$ and that $\mathbb{V}_{-\Delta}\left(\lambda_{i_{0}}\right)$ is a nontrivial $S O(2)$-representation,

(3) there exists $\lambda_{i_{0}} \in \sigma(-\Delta ; \Omega)$ such that

(a) $f^{\prime}(z)<\lambda_{i_{0}}<f^{\prime}\left(z_{0}\right)$ for all $z \in\left(Z_{+} \cup\{\infty\}\right) \backslash\left\{z_{0}\right\}$,

(b) there exists $k^{\prime} \in \mathbb{N}$ such that

(i) $\mathbb{V}_{-\Delta}\left(\lambda_{i_{0}}\right)=\mathbb{R}\left[1, k^{\prime}\right] \oplus \mathbb{R}\left[1, k^{\prime}\right]^{\perp}$

(ii) $\mathbb{R}\left[1, k^{\prime}\right] \not \subset \mathbb{V}_{-\Delta}\left(\lambda_{i}\right)$ for $\lambda_{i} \in \sigma(-\Delta ; \Omega) \cap\left(-\infty, \lambda_{i_{0}}\right)$.

Then there exists at least one nonconstant solution of equation (4.1.1).

Proof. The proof is similar to that of Theorem 4.1.2. By the properties of the degree for $S O(2)$-equivariant gradient maps we obtain

$$
\begin{gathered}
\nabla_{S O(2)}-\operatorname{deg}\left(\nabla \Phi, B_{\gamma_{\infty}}\left(H^{1}(\Omega)\right) \backslash \bigcup_{z \in Z} D_{\gamma_{z}}\left(H^{1}(\Omega), z\right)\right)= \\
=\nabla_{S O(2)}-\operatorname{deg}\left(\nabla \Phi, B_{\gamma_{\infty}}\left(H^{1}(\Omega)\right)\right)-\sum_{z \in Z} \nabla_{S O(2)}-\operatorname{deg}\left(\nabla \Phi, B_{\gamma_{z}}\left(H^{1}(\Omega), z\right)\right) .
\end{gathered}
$$

It remains to prove that

$$
\nabla_{S O(2)}-\operatorname{deg}\left(\nabla \Phi, B_{\gamma_{\infty}}\left(H^{1}(\Omega)\right)\right) \neq \sum_{z \in Z} \nabla_{S O(2)}-\operatorname{deg}\left(\nabla \Phi, B_{\gamma_{z}}\left(H^{1}(\Omega), z\right)\right) .
$$

Suppose, contrary to our claim, that

$$
\nabla_{S O(2)}-\operatorname{deg}\left(\nabla \Phi, B_{\gamma_{\infty}}\left(H^{1}(\Omega)\right)\right)=\sum_{z \in Z} \nabla_{S O(2)}-\operatorname{deg}\left(\nabla \Phi, B_{\gamma_{z}}\left(H^{1}(\Omega), z\right)\right) .
$$


If $z \in Z_{-}$and $k \in \mathbb{N}$, then, by Lemma 4.1.3, we get $\nabla_{S O(2)}-\operatorname{deg}_{\mathbb{Z}_{k}}\left(\nabla \Phi, B_{\gamma_{z}}\left(H^{1}(\Omega), z\right)\right)=0$ and $\nabla_{S O(2)}-\operatorname{deg}_{\mathbb{Z}_{k}}\left(\nabla \Phi, B_{\gamma_{\infty}}\left(H^{1}(\Omega)\right)\right)=\sum_{z \in Z_{+}} \nabla_{S O(2)}-\operatorname{deg}_{\mathbb{Z}_{k}}\left(\nabla \Phi, B_{\gamma_{z}}\left(H^{1}(\Omega), z\right)\right)$, which is equivalent to

$$
\begin{aligned}
& -\nabla_{S O(2)}-\operatorname{deg}_{\mathbb{Z}_{k}}\left(\nabla \Phi, B_{\gamma_{z_{0}}}\left(H^{1}(\Omega), z_{0}\right)\right)= \\
& =\sum_{z \in Z_{+} \backslash\left\{z_{0}\right\}} \nabla_{S O(2)}-\operatorname{deg}_{\mathbb{Z}_{k}}\left(\nabla \Phi, B_{\gamma_{z}}\left(H^{1}(\Omega), z\right)\right)-\nabla_{S O(2)}-\operatorname{deg}_{\mathbb{Z}_{k}}\left(\nabla \Phi, B_{\gamma_{\infty}}\left(H^{1}(\Omega)\right)\right) \text {. }
\end{aligned}
$$

Since $\nu\left(f^{\prime}(\infty)\right), \nu\left(f^{\prime}\left(z_{0}\right)\right)$ are even and Corollary 4.1.1, we have

$$
-\nabla_{S O(2)}-\operatorname{deg}_{\mathbb{Z}_{k^{\prime}}}\left(\nabla \Phi, B_{\gamma_{\infty}}\left(H^{1}(\Omega)\right)\right) \leq 0,-\nabla_{S O(2)}-\operatorname{deg}_{\mathbb{Z}_{k^{\prime}}}\left(\nabla \Phi, B_{\gamma_{z_{0}}}\left(H^{1}(\Omega), z_{0}\right)\right) \leq 0 .
$$

Notice that, in view of Theorem 4.1.1, to complete the proof it is enough to consider the case

$$
\left\{z \in Z_{+}: \nu\left(f^{\prime}(z)\right) \text { is even }\right\}=\left\{z_{0}\right\} .
$$

(1) Let $z_{1}, z_{2} \neq \infty$. Since $\mathbb{V}_{-\Delta}\left(\lambda_{0}\right)$ is a nontrivial $S O(2)$-representation, there is $k^{\prime} \in$ $\mathbb{N}$ such that $\mathbb{V}_{-\Delta}\left(\lambda_{0}\right)=\mathbb{R}\left[1, k^{\prime}\right] \oplus \mathbb{R}\left[1, k^{\prime}\right]^{\perp}$. Taking into account that $f^{\prime}\left(z_{1}\right), f^{\prime}\left(z_{2}\right)>$ $\lambda_{0}, \nu\left(f^{\prime}\left(z_{1}\right)\right), \nu\left(f^{\prime}\left(z_{1}\right)\right)$ are odd and Corollary 4.1.1 we obtain

$$
\nabla_{S O(2)}-\operatorname{deg}_{\mathbb{Z}_{k^{\prime}}}\left(\nabla \Phi, B_{\gamma_{z_{1}}}\left(H^{1}(\Omega), z_{1}\right)\right), \nabla_{S O(2)}-\operatorname{deg}_{\mathbb{Z}_{k^{\prime}}}\left(\nabla \Phi, B_{\gamma_{z_{2}}}\left(H^{1}(\Omega), z_{2}\right)\right)<0 .
$$

Since $f^{\prime}\left(z_{1}\right)>f^{\prime}\left(z_{0}\right)$, it follows that $\mathbb{V}\left(f^{\prime}\left(z_{0}\right)\right) \subset \mathbb{V}\left(f^{\prime}\left(z_{1}\right)\right)$ and consequently by Lemmas 2.1, 4.1.3, 4.1.4 we obtain

$$
\begin{aligned}
& \nabla_{S O(2)}-\operatorname{deg}_{\mathbb{Z}_{k^{\prime}}}\left(\nabla \Phi, B_{\gamma_{z_{1}}}\left(H^{1}(\Omega), z_{1}\right)\right)=\nabla_{S O(2)}-\operatorname{deg}_{\mathbb{Z}_{k^{\prime}}}\left(\nabla^{2} \Phi\left(z_{1}\right), B_{\gamma_{z_{1}}}\left(H^{1}(\Omega), z_{1}\right)\right)= \\
= & \nabla_{S O(2)}-\operatorname{deg}_{\mathbb{Z}_{k^{\prime}}}\left(-I d, B_{\gamma_{z_{1}}}\left(\mathbb{V}\left(f^{\prime}\left(z_{1}\right)\right)\right) \leq-\nabla_{S O(2)}-\operatorname{deg}_{\mathbb{Z}_{k^{\prime}}}\left(-I d, B_{\gamma_{z_{0}}}\left(\mathbb{V}\left(f^{\prime}\left(z_{0}\right)\right)\right)\right)=\right. \\
= & -\nabla_{S O(2)}-\operatorname{deg}_{\mathbb{Z}_{k^{\prime}}}\left(\nabla^{2} \Phi\left(z_{0}\right), B_{\gamma_{z_{0}}}\left(H^{1}(\Omega), z_{0}\right)\right)=-\nabla_{S O(2)}-\operatorname{deg}_{\mathbb{Z}_{k^{\prime}}}\left(\nabla \Phi, B_{\gamma_{z_{0}}}\left(H^{1}(\Omega), z_{0}\right)\right) .
\end{aligned}
$$

Taking into account (4.1.11), (4.1.12), Corollary 4.1.1 and the above inequalities we obtain

$$
\begin{gathered}
-\nabla_{S O(2)}-\operatorname{deg}_{\mathbb{Z}_{k^{\prime}}}\left(\nabla \Phi, B_{\gamma_{z_{0}}}\left(H^{1}(\Omega), z_{0}\right)\right) \geq \nabla_{S O(2)}-\operatorname{deg}_{\mathbb{Z}_{k^{\prime}}}\left(\nabla \Phi, B_{\gamma_{z_{1}}}\left(H^{1}(\Omega), z_{1}\right)\right)> \\
>\nabla_{S O(2)}-\operatorname{deg}_{\mathbb{Z}_{k^{\prime}}}\left(\nabla \Phi, B_{\gamma_{z_{2}}}\left(H^{1}(\Omega), z_{2}\right)\right)+\nabla_{S O(2)}-\operatorname{deg}_{\mathbb{Z}_{k^{\prime}}}\left(\nabla \Phi, B_{\gamma_{z_{1}}}\left(H^{1}(\Omega), z_{1}\right) \geq\right. \\
\geq \sum_{z \in Z \backslash\left\{z_{0}\right\}} \nabla_{S O(2)}-\operatorname{deg}_{\mathbb{Z}_{k^{\prime}}}\left(\nabla \Phi, B_{\gamma_{z}}\left(H^{1}(\Omega), z\right)\right)-\nabla_{S O(2)}-\operatorname{deg}_{\mathbb{Z}_{k}}\left(\nabla \Phi, B_{\gamma_{\infty}}\left(H^{1}(\Omega)\right)\right)= \\
=-\nabla_{S O(2)}-\operatorname{deg}_{\mathbb{Z}_{k^{\prime}}}\left(\nabla \Phi, B_{\gamma_{z_{0}}}\left(H^{1}(\Omega), z_{0}\right)\right),
\end{gathered}
$$

a contradiction. The same proof works for $z_{1}=\infty$ or $z_{2}=\infty$. The details are left to the reader.

(2) Assume that $z_{1} \neq \infty$. Since $\mathbb{V}_{-\Delta}\left(\lambda_{i_{0}}\right)$ is a nontrivial $S O(2)$-representation, there is $k^{\prime} \in \mathbb{N}$ such that $\mathbb{V}_{-\Delta}\left(\lambda_{i_{0}}\right)=\mathbb{R}\left[1, k^{\prime}\right] \oplus \mathbb{R}\left[1, k^{\prime}\right]^{\perp}$. Fix $z \in(Z \cup\{\infty\}) \backslash\left\{z_{0}, z_{1}\right\}$. Since $f^{\prime}(z)<\lambda_{0}, \mathbb{V}\left(f^{\prime}(z)\right)$ is a trivial $S O(2)$-representation and by Lemmas [2.1, 4.1.3] we have $\nabla_{S O(2)}-\operatorname{deg}_{\mathbb{Z}_{k^{\prime}}}\left(\nabla \Phi, B_{\gamma_{z}}\left(H^{1}(\Omega), z\right)\right)=0$. Thus

$$
-\nabla_{S O(2)}-\operatorname{deg}_{\mathbb{Z}_{k^{\prime}}}\left(\nabla \Phi, B_{\gamma_{z_{0}}}\left(H^{1}(\Omega), z_{0}\right)\right)=\sum_{z \in Z_{+} \backslash\left\{z_{0}\right\}} \nabla_{S O(2)}-\operatorname{deg}_{\mathbb{Z}_{k^{\prime}}}\left(\nabla \Phi, B_{\gamma_{z}}\left(H^{1}(\Omega), z\right)\right)-
$$




$$
-\nabla_{S O(2)}-\operatorname{deg}_{\mathbb{Z}_{k}}\left(\nabla \Phi, B_{\gamma_{\infty}}\left(H^{1}(\Omega)\right)\right)=\nabla_{S O(2)}-\operatorname{deg}_{\mathbb{Z}_{k^{\prime}}}\left(\nabla \Phi, B_{\gamma_{z_{1}}}\left(H^{1}(\Omega), z_{1}\right)\right) .
$$

Let $j_{0}, j_{1} \in \mathbb{N}$ be the largest integers such that

$$
\mathbb{V}\left(f^{\prime}\left(z_{0}\right)\right)=\mathbb{R}\left[j_{0}, k^{\prime}\right] \oplus \mathbb{R}\left[j_{0}, k^{\prime}\right]^{\perp}, \text { and } \mathbb{V}\left(f^{\prime}\left(z_{1}\right)\right)=\mathbb{R}\left[j_{1}, k^{\prime}\right] \oplus \mathbb{R}\left[j_{1}, k^{\prime}\right]^{\perp} .
$$

Since $f^{\prime}\left(z_{1}\right)<\lambda_{i_{0}}<f^{\prime}\left(z_{0}\right)$, we obtain $j_{1}<j_{0}$. Finally, by Lemmas 2.1, 4.1.3, we obtain

$$
-\nabla_{S O(2)}-\operatorname{deg}_{\mathbb{Z}_{k^{\prime}}}\left(\nabla \Phi, B_{\gamma_{z_{0}}}\left(H^{1}(\Omega), z_{0}\right)\right) \neq \nabla_{S O(2)}-\operatorname{deg}_{\mathbb{Z}_{k^{\prime}}}\left(\nabla \Phi, B_{\gamma_{z_{1}}}\left(H^{1}(\Omega), z_{1}\right)\right),
$$

a contradiction. The same proof remains valid if $z_{1}=\infty$ or $f^{\prime}\left(z_{0}\right)<\lambda_{i_{0}}<f^{\prime}\left(z_{1}\right)$. The details are left to the reader.

(3) Since $\mathbb{R}\left[1, k^{\prime}\right] \not \subset \mathbb{V}\left(f^{\prime}(z)\right)$ for every $z \in\left(Z_{+} \cup\{\infty\}\right) \backslash\left\{z_{0}\right\}$ and Lemmas 2.1, 4.1.3. $\nabla_{S O(2)}-\operatorname{deg}_{\mathbb{Z}_{k^{\prime}}}\left(\nabla \Phi, B_{\gamma_{z}}\left(H^{1}(\Omega), z\right)\right)=\nabla_{S O(2)}-\operatorname{deg}_{\mathbb{Z}_{k^{\prime}}}\left(-I d, B_{\gamma_{z}}\left(\mathbb{V}\left(f^{\prime}(z)\right)\right)=0\right.$, for every $z \in\left(Z_{+} \cup\{\infty\}\right) \backslash\left\{z_{0}\right\}$. Thus, by the above and (4.1.11), we obtain

$$
\begin{gathered}
-\nabla_{S O(2)}-\operatorname{deg}_{\mathbb{Z}_{k^{\prime}}}\left(\nabla \Phi, B_{\gamma_{z_{0}}}\left(H^{1}(\Omega), z_{0}\right)\right)= \\
=\sum_{z \in Z_{+} \backslash\left\{z_{0}\right\}} \nabla_{S O(2)}-\operatorname{deg}_{\mathbb{Z}_{k^{\prime}}}\left(\nabla \Phi, B_{\gamma_{z}}\left(H^{1}(\Omega), z\right)\right)-\nabla_{S O(2)}-\operatorname{deg}_{\mathbb{Z}_{k^{\prime}}}\left(\nabla \Phi, B_{\gamma_{\infty}}\left(H^{1}(\Omega)\right)\right)=0 .
\end{gathered}
$$

Since $\mathbb{V}_{-\Delta}\left(\lambda_{i_{0}}\right) \subset \mathbb{V}\left(f^{\prime}\left(z_{0}\right)\right), \mathbb{R}\left[1, k^{\prime}\right] \subset \mathbb{V}\left(f^{\prime}\left(z_{0}\right)\right)$ and consequently

$$
\nabla_{S O(2)}-\operatorname{deg}_{\mathbb{Z}_{k^{\prime}}}\left(\nabla \Phi, B_{\gamma_{z_{0}}}\left(H^{1}(\Omega), z_{0}\right)\right)=\nabla_{S O(2)}-\operatorname{deg}_{\mathbb{Z}_{k^{\prime}}}\left(-I d, B_{\gamma_{z_{0}}}\left(\mathbb{V}\left(f^{\prime}\left(z_{0}\right)\right)\right)\right) \neq 0,
$$

a contradiction.

Remark 4.1.3. Notice that in Theorems 4.1.2 4.1.4 the degree for $S O(2)$-equivariant gradient maps can not be replaced with the Leray-Schauder degree, since it vanishes. In fact, under assumptions of these theorems it can happen that

$$
\operatorname{deg}_{\mathrm{LS}}\left(\nabla \Phi, B_{\gamma_{\infty}}\left(H^{1}(\Omega)\right)\right)-\sum_{z \in Z} \operatorname{deg}_{L S}\left(\nabla \Phi, B_{\gamma_{z}}\left(H^{1}(\Omega), z\right)\right)=0 \in \mathbb{Z} .
$$

and that

$$
\nabla_{S O(2)}-\operatorname{deg}\left(\nabla \Phi, B_{\gamma_{\infty}}\left(H^{1}(\Omega)\right)\right)-\sum_{z \in Z} \nabla_{S O(2)}-\operatorname{deg}\left(\nabla \Phi, B_{\gamma_{z}}\left(H^{1}(\Omega), z\right)\right) \neq \Theta \in U(S O(2)) .
$$

In other words we obtain the existence of nonconstant solution of equation (4.1.1) in the situation when the Leray-Schauder degree is not applicable i.e. the assumptions of Theorem 4.1.1 are not fulfilled.

In the rest of this section we consider a degenerate case i.e. we allow $f^{\prime}\left(z_{0}\right) \in \sigma(-\Delta ; \Omega)$ for some $z_{0} \in Z \cup\{\infty\}$. To compute a local index of a degenerate isolated critical point of $\Phi$ we combine the splitting lemmas (Lemmas 3.2, 3.3 of [9]) and the product formula for the degree for $S O(2)$-equivariant gradient maps, Theorem 2.2 The following lemma is a consequence of splitting lemmas of 9 .

Lemma 4.1.5. Assume that assumptions (A.1), (A.5) are satisfied. Fix $z_{0} \in Z \cup\{\infty\}$ such that $f^{\prime}\left(z_{0}\right) \in \sigma(-\Delta ; \Omega)$ and $z_{0} \in H^{1}(\Omega)$ is an isolated critical point of $\Phi$. Then there exist $\alpha_{0}>0$ and $\varphi \in C_{S O(2)}^{2}\left(\mathbb{V}_{-\Delta}\left(f^{\prime}\left(z_{0}\right)\right), \mathbb{R}\right)$ such that $0 \in \mathbb{V}_{-\Delta}\left(f^{\prime}\left(z_{0}\right)\right)$ is an isolated critical point of $\varphi$ and that

$$
\nabla_{S O(2)}-\operatorname{deg}\left(\nabla \Phi, B_{\gamma_{z_{0}}}\left(H^{1}(\Omega), z_{0}\right)\right)=
$$




$$
=\nabla_{S O(2)}-\operatorname{deg}\left(\nabla \varphi, B_{\alpha_{0}}\left(\mathbb{V}_{-\Delta}\left(f^{\prime}\left(z_{0}\right)\right)\right)\right) \star \prod_{\lambda_{i}<f^{\prime}\left(z_{0}\right)} \nabla_{S O(2)}-\operatorname{deg}\left(-I d, B_{\alpha_{0}}\left(\mathbb{V}_{-\Delta}\left(\lambda_{i}\right)\right)\right) .
$$

Proof. Fix $z_{0} \in Z$ and remind that $\mathcal{K}: H^{1}(\Omega) \rightarrow H^{1}(\Omega)$ is an $S O(2)$-equivariant, selfadjoint, compact operator such that $\langle\mathcal{K} u, v\rangle_{H^{1}(\Omega)}=\int_{\Omega} u(x) v(x) d x$. Set $L=\left(1+f^{\prime}\left(z_{0}\right)\right) \mathcal{K}$, $\mathbb{V}_{0}=\operatorname{ker} I d-L$ and $W_{0}=\mathbb{V}_{0}^{\perp}=\operatorname{im} I d-L$. It is easy to see that $\nabla^{2} \Phi\left(z_{0}\right)=I d-L$ and $\mathbb{V}_{0}=\mathbb{V}_{-\Delta}\left(f^{\prime}\left(z_{0}\right)\right)$. Define $\nabla \eta_{0}: H^{1}(\Omega) \rightarrow H^{1}(\Omega), \nabla \eta_{0}=\nabla \Phi-(I d-L)$. Then $\nabla \eta_{0}$ is a compact, $S O(2)$-equivariant operator and $\left|\nabla \eta_{0}(u)\right|=o(|u|)$ as $|u| \rightarrow 0$. Now applying Lemma 3.2 of [9] we obtain $\alpha_{0}>0$ and $\varphi \in C_{S O(2)}^{2}\left(\mathbb{V}_{-\Delta}\left(f^{\prime}\left(z_{0}\right)\right), \mathbb{R}\right)$ with isolated critical point at the origin and such that

$$
\left.\nabla_{S O(2)}-\operatorname{deg}\left(\nabla \Phi, B_{\alpha_{0}}\left(H^{1}(\Omega)\right)\right)=\nabla_{S O(2)}-\operatorname{deg}\left(\left(\nabla \varphi,\left(I d-L_{0}\right)_{\mid W_{0}}\right), B_{\alpha_{0}}\left(\mathbb{V}_{0}\right)\right) \times B_{\alpha_{0}}\left(W_{0}\right)\right) .
$$

Finally, combining Theorem 2.2 with a slightly modified version of Lemma 3.2 (instead of the operator $\nabla_{u} \Psi(\cdot, \lambda)$ it is enough to consider the operator $\left.\nabla^{2} \Phi\left(z_{0}\right)_{\mid W_{0}}\right)$, we obtain

$$
\begin{gathered}
\left.\nabla_{S O(2)}-\operatorname{deg}\left(\left(\nabla \varphi,\left(I d-L_{0}\right)_{\mid W_{0}}\right), B_{\alpha_{0}}\left(\mathbb{V}_{0}\right)\right) \times B_{\alpha_{0}}\left(W_{0}\right)\right)= \\
=\nabla_{S O(2)}-\operatorname{deg}\left(\nabla \varphi, B_{\gamma_{z_{0}}}\left(\mathbb{V}_{-\Delta}\left(f^{\prime}\left(z_{0}\right)\right)\right)\right) \star \nabla_{S O(2)}-\operatorname{deg}\left(\left(I d-L_{0}\right)_{\mid W_{0}}, B_{\gamma_{z_{0}}}\left(W_{0}\right)\right)= \\
=\nabla_{S O(2)}-\operatorname{deg}\left(\nabla \varphi, B_{\gamma_{z_{0}}}\left(\mathbb{V}_{-\Delta}\left(f^{\prime}\left(z_{0}\right)\right)\right) \star \prod_{\lambda_{i}<f^{\prime}\left(z_{0}\right)} \nabla_{S O(2)}-\operatorname{deg}\left(-I d, B_{\gamma_{z_{0}}}\left(\mathbb{V}_{-\Delta}\left(\lambda_{i}\right)\right)\right),\right.
\end{gathered}
$$

which completes the proof.

The same proof remains valid for $z_{0}=\infty$ but instead of Lemma 3.2 of [9] we must use Lemma 3.3 of 9 . The details are left to the reader.

Corollary 4.1.2. Fix $z_{0} \in Z \cup\{\infty\}$ satisfying assumptions of Lemma 4.1.5 and $k^{\prime} \in \mathbb{N}$. Assume that

(1) $\mathbb{R}\left[1, k^{\prime}\right] \not \subset \mathbb{V}\left(f^{\prime}\left(z_{0}\right)\right)$

(2) $\mathbb{V}_{-\Delta}\left(f^{\prime}\left(z_{0}\right)\right)_{\mathbb{Z}_{k^{\prime}}}=\emptyset$.

Then $\nabla_{S O(2)}-\operatorname{deg}_{\mathbb{Z}_{k^{\prime}}}\left(\nabla \Phi, B_{\gamma_{z_{0}}}\left(H^{1}(\Omega), z_{0}\right)\right)=0$.

Proof. Take $\alpha_{0}>0$ and $\nabla \varphi$ as in Lemma 4.1.5. Then

$$
\begin{gathered}
\nabla_{S O(2)}-\operatorname{deg}\left(\nabla \Phi, B_{\gamma_{z_{0}}}\left(H^{1}(\Omega), z_{0}\right)\right)= \\
=\nabla_{S O(2)}-\operatorname{deg}\left(\nabla \varphi, B_{\alpha_{0}}\left(\mathbb{V}_{-\Delta}\left(f^{\prime}\left(z_{0}\right)\right)\right)\right) \star \nabla_{S O(2)}-\operatorname{deg}\left(-I d, B_{\alpha_{0}}\left(\mathbb{V}\left(f^{\prime}\left(z_{0}\right)\right)\right)\right) .
\end{gathered}
$$

By (1) and Lemma 2.1 we have $\nabla_{S O(2)}-\operatorname{deg}_{\mathbb{Z}_{k^{\prime}}}\left(-I d, B_{\alpha_{0}}\left(\mathbb{V}\left(f^{\prime}\left(z_{0}\right)\right)\right)\right)=0$. By (2) and Remark 2.3 we obtain $\nabla_{S O(2)}-\operatorname{deg}_{\mathbb{Z}_{k^{\prime}}}\left(\nabla \varphi, B_{\alpha_{0}}\left(\mathbb{V}_{-\Delta}\left(f^{\prime}\left(z_{0}\right)\right)\right)\right)=0$. The rest of the proof is a direct consequence of product formula (2.2).

Corollary 4.1.3. Fix $z_{0} \in Z \cup\{\infty\}$ satisfying assumptions of Lemma 4.1.5. If moreover, $\mathbb{V}_{-\Delta}\left(f^{\prime}\left(z_{0}\right)\right)^{S O(2)}=\{0\}$, then

$$
\nabla_{S O(2)}-\operatorname{deg}_{\mathbb{Z}_{k}}\left(\nabla \Phi, B_{\gamma_{z_{0}}}\left(H^{1}(\Omega), z_{0}\right)\right)=\nabla_{S O(2)}-\operatorname{deg}_{\mathbb{Z}_{k}}\left(-I d, B_{\gamma_{z_{0}}}\left(\mathbb{V}\left(f^{\prime}\left(z_{0}\right)\right)\right)\right),
$$

for all $k \in \mathbb{N}$ such that $\mathbb{V}_{-\Delta}\left(f^{\prime}\left(z_{0}\right)\right)_{\mathbb{Z}_{k}}=\emptyset$. 
Proof. Take $\alpha_{0}>0$ and $\nabla \varphi$ as in Lemma 4.1.5, Then

$$
\begin{gathered}
\nabla_{S O(2)}-\operatorname{deg}\left(\nabla \Phi, B_{\gamma_{z_{0}}}\left(H^{1}(\Omega), z_{0}\right)\right)= \\
=\nabla_{S O(2)}-\operatorname{deg}\left(\nabla \varphi, B_{\alpha_{0}}\left(\mathbb{V}_{-\Delta}\left(f^{\prime}\left(z_{0}\right)\right)\right)\right) \star \nabla_{S O(2)}-\operatorname{deg}\left(-I d, B_{\alpha_{0}}\left(\mathbb{V}\left(f^{\prime}\left(z_{0}\right)\right)\right)\right) .
\end{gathered}
$$

Since $\mathbb{V}_{-\Delta}\left(f^{\prime}\left(z_{0}\right)\right)^{S O(2)}=\{0\}, \nabla_{S O(2)}-\operatorname{deg}_{S O(2)}\left(\nabla \varphi, B_{\gamma_{z_{0}}}\left(\mathbb{V}_{-\Delta}\left(f^{\prime}\left(z_{0}\right)\right), 0\right)\right)=1$. Moreover, since $\mathbb{V}_{-\Delta}\left(f^{\prime}\left(z_{0}\right)\right)_{\mathbb{Z}_{k}}=\emptyset$ and Remark 2.3. $\nabla_{S O(2)}-\operatorname{deg}_{\mathbb{Z}_{k}}\left(\nabla \varphi, B_{\gamma_{z_{0}}}\left(\mathbb{V}_{-\Delta}\left(f^{\prime}\left(z_{0}\right)\right), 0\right)\right)=0$. The rest of the proof is a direct consequence of formula (2.2).

We can now proof the analog of Theorems 4.1.2, 4.1.3, 4.1.4. It is worth to point out that in this theorem we allow $\bigcup_{z \in Z \cup\{\infty\}}\left\{f^{\prime}(z)\right\} \cap \sigma(-\Delta ; \Omega) \neq \emptyset$.

Theorem 4.1.5. Let assumptions (A.1)-(A.3), (A.5) be fulfilled. Moreover, assume that there are $z_{0} \in Z \cup\{\infty\}, \lambda_{i_{0}} \in \sigma(-\Delta ; \Omega)$ and $k^{\prime} \in \mathbb{N}$ such that

(1) either $f^{\prime}\left(z_{0}\right) \notin \sigma(-\Delta ; \Omega)$ or $\mathbb{V}_{-\Delta}\left(f^{\prime}\left(z_{0}\right)\right)^{S O(2)}=\{0\}$ and $\mathbb{V}_{-\Delta}\left(f^{\prime}\left(z_{0}\right)\right)_{\mathbb{Z}_{k^{\prime}}}=\emptyset$,

(2) $f^{\prime}\left(z_{0}\right)>\lambda_{i_{0}}>f^{\prime}(z)$ for all $z \in(Z \cup\{\infty\}) \backslash\left\{z_{0}\right\}$,

(3) $\mathbb{R}\left[1, k^{\prime}\right] \subset \mathbb{V}_{-\Delta}\left(\lambda_{i_{0}}\right)$,

(4) $\mathbb{V}_{-\Delta}\left(\lambda_{i}\right)_{\mathbb{Z}_{k^{\prime}}}=\emptyset$ for all $\lambda_{i} \in \sigma(-\Delta ; \Omega), \lambda_{i}<\lambda_{i_{0}}$.

Then there exists at least one nonconstant solution of equation (4.1.1).

Proof. Without loss of generality we can assume that elements of $Z \cup\{\infty\}$ are isolated critical points of the potential $\Phi$. To complete the proof it is enough to show that

$$
\nabla_{S O(2)}-\operatorname{deg}\left(\nabla \Phi, B_{\gamma_{\infty}}\left(H^{1}(\Omega)\right)\right) \neq \sum_{z \in Z} \nabla_{S O(2)}-\operatorname{deg}\left(\nabla \Phi, B_{\gamma_{z}}\left(H^{1}(\Omega), z\right)\right) .
$$

Suppose, contrary to our claim, that

$$
\nabla_{S O(2)}-\operatorname{deg}\left(\nabla \Phi, B_{\gamma_{\infty}}\left(H^{1}(\Omega)\right)\right)=\sum_{z \in Z} \nabla_{S O(2)}-\operatorname{deg}\left(\nabla \Phi, B_{\gamma_{z}}\left(H^{1}(\Omega), z\right)\right) .
$$

Fix $z \in(Z \cup\{\infty\}) \backslash\left\{z_{0}\right\}$. By assumptions (2), (4) we obtain that $\mathbb{V}_{-\Delta}\left(\lambda_{i}\right)_{Z_{k^{\prime}}}=\emptyset$ for all $\lambda_{i} \in \sigma(-\Delta ; \Omega) \cap\left(-\infty, f^{\prime}(z)\right]$. Therefore $\mathbb{R}\left[1, k^{\prime}\right] \not \subset \mathbb{V}\left(f^{\prime}(z)\right)$ and if $f^{\prime}(z) \in \sigma(-\Delta ; \Omega)$, then $\mathbb{V}_{-\Delta}\left(f^{\prime}(z)\right)_{\mathbb{Z}_{k^{\prime}}}=\emptyset$. Hence, from Corollary 4.1.2, we obtain

$$
\nabla_{S O(2)}-\operatorname{deg}_{Z_{k^{\prime}}}\left(\nabla \Phi, B_{\gamma_{z}}\left(H^{1}(\Omega), z\right)\right)=0,
$$

for all $z \in(Z \cup\{\infty\}) \backslash\left\{z_{0}\right\}$.

If $f^{\prime}\left(z_{0}\right) \notin \sigma(-\Delta ; \Omega)$, then, by Lemmas 4.1.3 $\left(z_{0} \in Z\right)$ or Lemma 4.1.4 $\left(z_{0}=\infty\right)$, we get

$$
\nabla_{S O(2)}-\operatorname{deg}_{Z_{k^{\prime}}}\left(\nabla \Phi, B_{\gamma_{z_{0}}}\left(H^{1}(\Omega), z_{0}\right)\right)=\nabla_{S O(2)}-\operatorname{deg}_{Z_{k^{\prime}}}\left(-I d, B_{\gamma_{z_{0}}}\left(\mathbb{V}\left(f^{\prime}\left(z_{0}\right)\right), z_{0}\right)\right) .
$$

If $f^{\prime}\left(z_{0}\right) \in \sigma(-\Delta ; \Omega)$, then, by assumption (1) and Corollary 4.1.3. we get

$$
\nabla_{S O(2)}-\operatorname{deg}_{Z_{k^{\prime}}}\left(\nabla \Phi, B_{\gamma_{z_{0}}}\left(H^{1}(\Omega), z_{0}\right)\right)=\nabla_{S O(2)}-\operatorname{deg}_{Z_{k^{\prime}}}\left(-I d, B_{\gamma_{z_{0}}}\left(\mathbb{V}\left(f^{\prime}\left(z_{0}\right)\right), z_{0}\right)\right) .
$$

Finally, since $\lambda_{i_{0}}<f^{\prime}\left(z_{0}\right)$ and $\mathbb{R}\left[1, k^{\prime}\right] \subset \mathbb{V}_{-\Delta}\left(\lambda_{i_{0}}\right)$, we obtain $\mathbb{R}\left[1, k^{\prime}\right] \subset \mathbb{V}\left(f^{\prime}\left(z_{0}\right)\right)$. Thus, by Lemma 2.1 , we obtain $\nabla_{S O(2)}-\operatorname{deg}_{Z_{k^{\prime}}}\left(-I d, B_{\gamma_{z_{0}}}\left(\mathbb{V}\left(f^{\prime}\left(z_{0}\right)\right), z_{0}\right)\right) \neq 0$ and consequently

$$
\nabla_{S O(2)}-\operatorname{deg}_{Z_{k^{\prime}}}\left(\nabla \Phi, B_{\gamma_{z_{0}}}\left(H^{1}(\Omega), z_{0}\right)\right) \neq 0
$$


Combining (4.1.14) with (4.1.15) we get

$$
\begin{gathered}
\nabla_{S O(2)}-\operatorname{deg}\left(\nabla \Phi, B_{\gamma_{\infty}}\left(H^{1}(\Omega), 0\right)\right)-\sum_{z \in Z} \nabla_{S O(2)}-\operatorname{deg}\left(\nabla \Phi, B_{\gamma_{z}}\left(H^{1}(\Omega), z\right)\right)= \\
=-\nabla_{S O(2)}-\operatorname{deg}\left(\nabla \Phi, B_{\gamma_{z_{0}}}\left(H^{1}(\Omega), z_{0}\right)\right) \neq 0,
\end{gathered}
$$

contrary to (4.1.13).

Remark 4.1.4. Let us notice that in the above theorem the same proof works for assumption (4) replaced by assumptions

(1) $\mathbb{R}\left[1, k^{\prime}\right] \not \subset \mathbb{V}_{-\Delta}\left(\lambda_{i}\right)$ for all $\lambda_{i} \in \sigma(-\Delta ; \Omega) \cap\left(-\infty, \lambda_{i_{0}}\right)$,

(2) $\mathbb{V}_{-\Delta}\left(f^{\prime}(z)\right)_{\mathbb{Z}_{k^{\prime}}}=\emptyset$ for all $z \in(Z \cup\{\infty\}) \cap \sigma(-\Delta ; \Omega)$.

The details are left to the reader.

4.2. Continuation of solutions. Consider a family of equations of the form

$$
\left\{\begin{aligned}
-\Delta u & =f(u, \lambda) & & \text { in } \Omega, \\
\frac{\partial u}{\partial \nu} & =0 & & \text { on } \partial \Omega,
\end{aligned}\right.
$$

where $f \in C^{1}(\mathbb{R} \times \mathbb{R}, \mathbb{R}), f(\cdot, \lambda)$ satisfies condition (A.1) for every $\lambda \in \mathbb{R}$ and $\Omega \subset \mathbb{R}^{n}$ is an open, bounded set with $C^{1-}$-boundary.

In this section we study continuation of nonconstant solutions of family (4.2.1).

Remark 4.2.1. Consider a functional $\Phi \in C^{2}\left(H^{1}(\Omega) \times \mathbb{R}, \mathbb{R}\right)$ defined as follows

$$
\Phi(u, \lambda)=\int_{\Omega}|\nabla u(x)|^{2}-F(u(x), \lambda) d x
$$

where $F_{u}^{\prime}=f$.

Define $Z_{0}=(f(\cdot, 0))^{-1}(0)$ and assume that

(1) $\# Z_{0}<\infty$,

(2) all the elements of $Z_{0} \cup\{\infty\}$ are isolated critical points of $\Phi(\cdot, 0)$.

Define an open bounded set $\mathcal{U}$ in the following way

$$
\mathcal{U}=B_{\gamma_{\infty}}\left(H^{1}(\Omega)\right) \backslash \bigcup_{z \in Z_{0}} D_{\gamma_{z}}\left(H^{1}(\Omega), z\right) .
$$

Since $\left(\nabla_{u} \Phi(\cdot, 0)\right)^{-1}(0) \cap \partial \mathcal{U}=\emptyset,\left(\nabla_{u} \Phi(\cdot, 0)\right)^{-1}(0) \cap \partial \mathcal{U}=\emptyset$. Therefore, by the properties of the Leray-Schauder degree, we obtain

$$
\begin{gathered}
\operatorname{deg}_{\mathrm{LS}}\left(\nabla_{u} \Phi(\cdot, 0), \mathcal{U}, 0\right)= \\
=\operatorname{deg}_{\mathrm{LS}}\left(\nabla_{u} \Phi(\cdot, 0), B_{\gamma_{\infty}}\left(H^{1}(\Omega)\right), 0\right)-\sum_{z \in Z_{0}} \operatorname{deg}_{\mathrm{LS}}\left(\nabla_{u} \Phi(\cdot, 0), B_{\gamma_{z}}\left(H^{1}(\Omega), z\right), 0\right) .
\end{gathered}
$$

If moreover, assumption (A.5) is fulfilled, then $\Phi \in C_{S O(2)}^{2}\left(H^{1}(\Omega) \times \mathbb{R}, \mathbb{R}\right)$ and $\mathcal{U}$ is $S O(2)$ invariant. Therefore, by the properties of the degree for $S O(2)$-equivariant gradient maps, we obtain

$$
\nabla_{S O(2)}-\operatorname{deg}\left(\nabla \Phi_{u}(\cdot, 0), \mathcal{U}\right)=
$$




$$
=\nabla_{S O(2)}-\operatorname{deg}\left(\nabla_{u} \Phi(\cdot, 0), B_{\gamma_{\infty}}\left(H^{1}(\Omega)\right)\right)-\sum_{z \in Z_{0}} \nabla_{S O(2)}-\operatorname{deg}\left(\nabla_{u} \Phi(\cdot, 0), B_{\gamma_{z}}\left(H^{1}(\Omega), z\right)\right) .
$$

Theorem 4.2.1. Fix $f \in C^{1}(\mathbb{R} \times \mathbb{R}, \mathbb{R})$ and assume that $f(\cdot, 0)$ satisfies assumptions of Theorem 4.1.1. Then there exist closed connected sets $\mathcal{C}^{ \pm}$such that

$$
\mathcal{C}^{-} \subset\left(H^{1}(\Omega) \times(-\infty, 0]\right) \cap\left(\nabla_{u} \Phi\right)^{-1}(0) \text { and } \mathcal{C}^{+} \subset\left(H^{1}(\Omega) \times[0,+\infty)\right) \cap\left(\nabla_{u} \Phi\right)^{-1}(0) .
$$

Moreover, for $\mathcal{C}=\mathcal{C}^{ \pm}$

(i) $\mathcal{C} \cap\left(\left(B_{\gamma_{\infty}}\left(H^{1}(\Omega)\right) \backslash \bigcup_{z \in Z_{0}} D_{\gamma_{z}}\left(H^{1}(\Omega), z\right)\right) \times\{0\}\right) \neq \emptyset$

(ii) either $\mathcal{C}$ is unbounded or $\mathcal{C} \cap\left(Z_{0} \times\{0\}\right) \neq \emptyset$.

Proof. Repeating the reasoning from the proof of Theorem 4.1.1 we obtain

$$
\operatorname{deg}_{\mathrm{LS}}\left(\nabla_{u} \Phi(\cdot, 0), B_{\gamma_{\infty}}\left(H^{1}(\Omega)\right), 0\right) \neq \sum_{z \in Z_{0}} \operatorname{deg}_{\mathrm{LS}}\left(\nabla_{u} \Phi(\cdot, 0), B_{\gamma_{z}}\left(H^{1}(\Omega), z\right), 0\right) .
$$

Define $\mathcal{U}=B_{\gamma_{\infty}}\left(H^{1}(\Omega)\right) \backslash \bigcup_{z \in Z_{0}} D_{\gamma_{z}}\left(H^{1}(\Omega), z\right)$ and notice that $\operatorname{deg}_{\mathrm{LS}}\left(\nabla_{u} \Phi(\cdot, 0), \mathcal{U}, 0\right) \neq 0$.

Applying Theorem 2.5 we obtain the existence of closed connected sets $\mathcal{C}^{ \pm}$such that

$$
\begin{aligned}
& \mathcal{C}^{-} \subset\left(H^{1}(\Omega) \times(-\infty, 0]\right) \cap\left(\nabla_{u} \Phi\right)^{-1}(0), \\
& \mathcal{C}^{+} \quad \subset\left(H^{1}(\Omega) \times[0,+\infty)\right) \cap\left(\nabla_{u} \Phi\right)^{-1}(0),
\end{aligned}
$$

$\mathcal{C}=\mathcal{C}^{ \pm}$satisfies (i) and either $\mathcal{C}$ is unbounded or else $C \cap\left(\left(H^{1}(\Omega) \backslash \operatorname{cl}(\mathcal{U})\right) \times\{0\}\right) \neq \emptyset$.

By definition $\left(H^{1}(\Omega) \backslash \operatorname{cl}(\mathcal{U})\right) \cap\left(\nabla_{u} \Phi(\cdot, 0)\right)^{-1}(0) \subset \bigcup_{z \in Z_{0}} B_{\gamma_{z}}\left(H^{1}(\Omega), z\right)$. On the other hand $\bigcup_{z \in Z_{0}} B_{\gamma_{z}}\left(H^{1}(\Omega), z\right) \cap\left(\nabla_{u} \Phi(\cdot, 0)\right)^{-1}(0)=Z_{0}$, which completes the proof.

Theorem 4.2.2. Fix $f \in C^{1}(\mathbb{R} \times \mathbb{R}, \mathbb{R})$ and assume that $f(\cdot, 0)$ satisfies assumptions of one of Theorems 4.1.2, 4.1.2, 4.1.4. Then there exist closed connected sets $\mathcal{C}^{ \pm}$such that

$$
\mathcal{C}^{-} \subset\left(H^{1}(\Omega) \times(-\infty, 0]\right) \cap\left(\nabla_{u} \Phi\right)^{-1}(0) \text { and } \mathcal{C}^{+} \subset\left(H^{1}(\Omega) \times[0,+\infty)\right) \cap\left(\nabla_{u} \Phi\right)^{-1}(0) .
$$

Moreover, for $\mathcal{C}=\mathcal{C}^{ \pm}$

(i) $\mathcal{C} \cap\left(\left(B_{\gamma_{\infty}}\left(H^{1}(\Omega)\right) \backslash \bigcup_{z \in Z_{0}} D_{\gamma_{z}}\left(H^{1}(\Omega), z\right)\right) \times\{0\}\right) \neq \emptyset$,

(ii) either $\mathcal{C}$ is unbounded or $\mathcal{C} \cap\left(Z_{0} \times\{0\}\right) \neq \emptyset$.

Proof. Repeating the reasoning from the proofs of Theorems 4.1.2 4.1.4 we obtain

$$
\nabla_{S O(2)}-\operatorname{deg}\left(\nabla_{u} \Phi(\cdot, 0), B_{\gamma_{\infty}}\left(H^{1}(\Omega)\right)\right) \neq \sum_{z \in Z_{0}} \nabla_{S O(2)}-\operatorname{deg}\left(\nabla_{u} \Phi(\cdot, 0), B_{\gamma_{z}}\left(H^{1}(\Omega), z\right)\right) .
$$

Set $\mathcal{U}=B_{\gamma_{\infty}}\left(H^{1}(\Omega)\right) \backslash \bigcup_{z \in Z_{0}} D_{\gamma_{z}}\left(H^{1}(\Omega), z\right)$. Notice that by Remark 4.2.1 we obtain $\nabla_{S O(2)}-\operatorname{deg}(\nabla \Phi(\cdot, 0), \mathcal{U}) \neq \Theta$. The rest of the proof is a direct consequence of Theorem 2.5 
Theorem 4.2.3. Assume that $f(\cdot, 0)$ satisfies assumptions of Theorem 4.1.5. Then there exists infinite sequence of nonconstant solutions of equation (4.2.1) with $\lambda=0$ converging to some $z \in Z_{0}$ or there exist closed connected sets $\mathcal{C}^{ \pm}$such that

$$
\mathcal{C}^{-} \subset\left(H^{1}(\Omega) \times(-\infty, 0]\right) \cap\left(\nabla_{u} \Phi\right)^{-1}(0) \text { and } \mathcal{C}^{+} \subset\left(H^{1}(\Omega) \times[0,+\infty)\right) \cap\left(\nabla_{u} \Phi\right)^{-1}(0) .
$$

Moreover, for $\mathcal{C}=\mathcal{C}^{ \pm}$

(i) $\mathcal{C} \cap\left(\left(B_{\gamma_{\infty}}\left(H^{1}(\Omega)\right) \backslash \bigcup_{z \in Z_{0}} D_{\gamma_{z}}\left(H^{1}(\Omega), z\right)\right) \times\{0\}\right) \neq \emptyset$,

(ii) either $\mathcal{C}$ is unbounded or $\mathcal{C} \cap\left(Z_{0} \times\{0\}\right) \neq \emptyset$.

Proof. Suppose that doesn't exist a sequence of nonconstant solutions of equation (4.2.1) with $\lambda=0$ converging to some point in $Z_{0}$. Then all the points $z \in Z_{0}$ are isolated critical points of $\Phi(\cdot, 0)$. Repeating the reasoning from the proof of Theorem 4.1.5 we obtain

$$
\nabla_{S O(2)}-\operatorname{deg}\left(\nabla_{u} \Phi(\cdot, 0), B_{\gamma_{\infty}}\left(H^{1}(\Omega)\right)\right) \neq \sum_{z \in Z_{0}} \nabla_{S O(2)}-\operatorname{deg}\left(\nabla_{u} \Phi(\cdot, 0), B_{\gamma_{z}}\left(H^{1}(\Omega), z\right)\right) .
$$

We set $\mathcal{U}=B_{\gamma_{\infty}}\left(H^{1}(\Omega)\right) \backslash \bigcup_{z \in Z_{0}} D_{\gamma_{z}}\left(H^{1}(\Omega), z\right)$. Applying Remark 4.2.1 we obtain the following $\nabla_{S O(2)}-\operatorname{deg}(\nabla \Phi, \mathcal{U}) \neq \Theta$. The rest of the proof is a direct consequence of Theorem 2.5.

4.3. Bifurcations from infinity. In this section we study bifurcations from infinity of solutions of a family of equations of the form

$$
\left\{\begin{aligned}
-\Delta u & =f(u, \lambda) & & \text { in } \Omega, \\
\frac{\partial u}{\partial \nu} & =0 & & \text { on } \partial \Omega,
\end{aligned}\right.
$$

where $f \in C^{1}(\mathbb{R} \times \mathbb{R}, \mathbb{R}), f(\cdot, \lambda)$ satisfies condition (A.1) for every $\lambda \in \mathbb{R} . \Omega \subset \mathbb{R}^{n}$ is an open, bounded set with $C^{1-}$-boundary. Moreover, we assume that assumption (A.5) is fulfilled.

(B.1) Fix $\lambda_{+}>\lambda_{-}$and assume that $f\left(\cdot, \lambda_{ \pm}\right)$satisfy assumption (A.2) and $f^{\prime}\left(\infty, \lambda_{ \pm}\right) \notin$ $\sigma(-\Delta ; \Omega)$.

Notice that under such an assumption $\nabla_{u}^{2} \Phi\left(\infty, \lambda_{ \pm}\right)=I d-f^{\prime}\left(\infty, \lambda_{ \pm}\right) \mathcal{K}$, where operator $\mathcal{K}: H^{1}(\Omega) \rightarrow H^{1}(\Omega)$ is given by the formula $\langle\mathcal{K} u, v\rangle_{H^{1}(\Omega)}=\int_{\Omega} u(x) v(x) d x$. Moreover, $\nabla_{u}^{2} \Phi\left(\infty, \lambda_{ \pm}\right)$is a linear isomorphism iff $f^{\prime}\left(\infty, \lambda_{ \pm}\right) \notin \sigma(-\Delta ; \Omega)$. Therefore operator $\nabla_{u} \Phi\left(\cdot, \lambda_{ \pm}\right)$is asymptotically linear at infinity and its derivative at infinity is a linear isomorphism. Thus there exists $\gamma>0$ such that

$$
\left(\nabla_{u} \Phi\left(\cdot, \lambda_{ \pm}\right)\right)^{-1}(0) \subset B_{\gamma}\left(H^{1}(\Omega)\right) \times\left\{\lambda_{ \pm}\right\}
$$

and we can define $\operatorname{BIF}\left(\infty,\left[\lambda_{-}, \lambda_{+}\right]\right) \in U(S O(2))$. The following theorem is a direct consequence of Theorem 2.6. 
Theorem 4.3.1. Let $\lambda_{+}>\lambda_{-}$satisfy assumption (B.1) and fix $\gamma>0$ such that condition (4.3.2) holds. If $\operatorname{BIF}\left(\infty,\left[\lambda_{-}, \lambda_{+}\right]\right) \neq \Theta \in U(S O(2))$, then there exists an unbounded closed connected component $\mathcal{C}$ of $\left(\nabla_{u} \Phi\right)^{-1}(0) \cap\left(H^{1}(\Omega) \times\left[\lambda_{-}, \lambda_{+}\right]\right)$such that $\mathcal{C} \cap\left(B_{\gamma}\left(H^{1}(\Omega)\right) \times\right.$ $\left.\left\{\lambda_{-}, \lambda_{+}\right\}\right) \neq \emptyset$.

In the next lemma show how to verify that the bifurcation index $\operatorname{BIF}\left(\infty,\left[\lambda_{-}, \lambda_{+}\right]\right) \in$ $U(S O(2))$ is nontrivial. In this lemma, for simplicity, we assume that $f^{\prime}\left(\infty, \lambda_{+}\right)>$ $f^{\prime}\left(\infty, \lambda_{-}\right)$. It is clear that similar lemma can be formulated if $f^{\prime}\left(\infty, \lambda_{+}\right)<f^{\prime}\left(\infty, \lambda_{-}\right)$.

Lemma 4.3.1. Let $\lambda_{+}>\lambda_{-}$satisfy assumption (B.1) and $f^{\prime}\left(\infty, \lambda_{+}\right)>f^{\prime}\left(\infty, \lambda_{-}\right)$. Then $\operatorname{BIF}\left(\infty,\left[\lambda_{-}, \lambda_{+}\right]\right) \neq \Theta \in U(S O(2))$ iff at least one of the following conditions is satisfied:

(1) there exists $\lambda_{i_{0}} \in \sigma(-\Delta ; \Omega)$, such that $f^{\prime}\left(\infty, \lambda_{-}\right)<\lambda_{i_{0}}<f^{\prime}\left(\infty, \lambda_{+}\right)$and $\mathbb{V}_{-\Delta}\left(\lambda_{i_{0}}\right) \neq \mathbb{V}_{-\Delta}\left(\lambda_{i_{0}}\right)^{S O(2)}$

$$
\sum_{f^{\prime}\left(\infty, \lambda_{-}\right)<\lambda_{i}<f^{\prime}\left(\infty, \lambda_{+}\right)} \operatorname{dim} \mathbb{V}_{-\Delta}\left(\lambda_{i}\right) \text { is odd. }
$$

Proof. Fix $\gamma>0$ such that condition (4.3.2) holds. Directly from the definition we have

$$
\begin{gathered}
\operatorname{BIF}\left(\infty,\left[\lambda_{-}, \lambda_{+}\right]\right)= \\
=\nabla_{S O(2)}-\operatorname{deg}\left(\nabla_{u} \Phi\left(\cdot, \lambda_{+}\right), B_{\gamma}(\mathbb{H})\right)-\nabla_{S O(2)}-\operatorname{deg}\left(\nabla_{u} \Phi\left(\cdot, \lambda_{-}\right), B_{\gamma}(\mathbb{H})\right) .
\end{gathered}
$$

By Lemma 4.1.4 we obtain

$$
\begin{gathered}
\operatorname{BIF}\left(\infty,\left[\lambda_{-}, \lambda_{+}\right]\right)=\left(\prod_{f^{\prime}\left(\infty, \lambda_{-}\right)<\lambda_{i}<f^{\prime}\left(\infty, \lambda_{+}\right)} \nabla_{S O(2)}-\operatorname{deg}\left(-I d, B_{\gamma_{\infty}}\left(\mathbb{V}_{-\Delta}\left(\lambda_{i}\right)\right)\right)-\mathbb{I}\right) \star \\
\star \nabla_{S O(2)}-\operatorname{deg}\left(\nabla_{u} \Phi\left(\cdot, \lambda_{-}\right), B_{\gamma}(\mathbb{H})\right) .
\end{gathered}
$$

Since $f^{\prime}\left(\infty, \lambda_{-}\right) \notin \sigma(-\Delta ; \Omega)$ and Lemma 4.1.4. $\nabla_{S O(2)}-\operatorname{deg}\left(\nabla_{u} \Phi\left(\cdot, \lambda_{-}\right), B_{\gamma}(\mathbb{H})\right)$ is the degree of isomorphism.

Thus, by Lemma 2.4, $\nabla_{S O(2)}-\operatorname{deg}_{S O(2)}\left(\nabla_{u} \Phi\left(\cdot, \lambda_{-}\right), B_{\gamma}(\mathbb{H})\right)= \pm 1$. Consequently by Remark 2.1 we obtain that $\nabla_{S O(2)}-\operatorname{deg}\left(\nabla_{u} \Phi\left(\cdot, \lambda_{-}\right), B_{\gamma}(\mathbb{H})\right)$ is invertible in $U(S O(2))$ and therefore $\operatorname{BIF}\left(\infty,\left[\lambda_{-}, \lambda_{+}\right]\right)=\Theta \in U(S O(2))$ iff

$$
\prod_{f^{\prime}\left(\infty, \lambda_{-}\right)<\lambda_{i}<f^{\prime}\left(\infty, \lambda_{+}\right)} \nabla_{S O(2)}-\operatorname{deg}\left(-I d, B_{\gamma_{\infty}}\left(\mathbb{V}_{-\Delta}\left(\lambda_{i}\right)\right)\right)=\mathbb{I} .
$$

This equality can be rewritten as follows

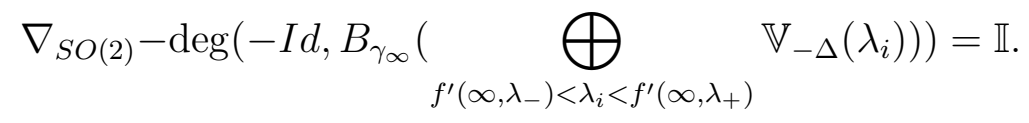

By Lemma 2.4 condition (4.3.3) is satisfied iff $\bigoplus \mathbb{V}_{-\Delta}\left(\lambda_{i}\right)$ is a trivial and even dimensional $S O(2)$-representation.

$$
f^{\prime}\left(\infty, \lambda_{-}\right)<\lambda_{i}<f^{\prime}\left(\infty, \lambda_{+}\right)
$$

Therefore, $\operatorname{BIF}\left(\infty,\left[\lambda_{-}, \lambda_{+}\right]\right) \neq \Theta \in U(S O(2))$ iff $\quad \bigoplus \quad \mathbb{V}_{-\Delta}\left(\lambda_{i}\right)$ is a non$f^{\prime}\left(\infty, \lambda_{-}\right)<\lambda_{i}<f^{\prime}\left(\infty, \lambda_{+}\right)$

trivial $S O(2)$-representation or is odd dimensional, which completes the proof. 
Corollary 4.3.1. Fix $\lambda_{ \pm} \in \mathbb{R}$ and $\eta>0$ as in Theorem 4.3.1. Additionally, assume that $f^{\prime}\left(\infty, \lambda_{+}\right)>f^{\prime}\left(\infty, \lambda_{-}\right)$and that one of the following conditions is satisfied:

(1) there exists $\lambda_{i_{0}} \in \sigma(-\Delta ; \Omega)$, such that $f^{\prime}\left(\infty, \lambda_{-}\right)<\lambda_{i_{0}}<f^{\prime}\left(\infty, \lambda_{+}\right)$and

$$
\text { (2) } \sum_{f^{\prime}\left(\infty, \lambda_{-}\right)<\lambda_{i}<f^{\prime}\left(\infty, \lambda_{+}\right)}^{\mathbb{V}_{-\Delta}\left(\lambda_{i_{0}}\right) \neq \mathbb{V}_{-\Delta}\left(\lambda_{i_{0}}\right)^{S O(2)}} \operatorname{dim} \mathbb{V}_{-\Delta}\left(\lambda_{i}\right) \text { is odd. }
$$

Then the statement of Theorem 4.3.1 holds true.

Remark 4.3.1. It is easy to see that the analogous corollary as above holds true also if $f^{\prime}\left(\infty, \lambda_{+}\right)<f^{\prime}\left(\infty, \lambda_{-}\right)$.

We will need the following assumption.

(B.2) Let $f(\cdot, \lambda)$ satisfy assumption (A.2) for all $\lambda \in \mathbb{R}$.

Notice that under assumption (B.2) $\nabla_{u} \Phi(\cdot, \lambda)$ is asymptotically linear for all $\lambda \in \mathbb{R}$. Hence $\nabla_{u}^{2} \Phi(\infty, \lambda)$ is defined for all $\lambda \in \mathbb{R}$ and $\nabla_{u}^{2} \Phi(\infty, \lambda)=I d-f^{\prime}(\infty, \lambda) \mathcal{K}$. Let $\lambda_{0} \in \mathbb{R}$ be such that $f^{\prime}\left(\infty, \lambda_{0}\right) \in \sigma(-\Delta ; \Omega)$. Choose $\epsilon>0$, define $\lambda_{ \pm}=\lambda_{0} \pm \epsilon$ and assume that

$$
\left\{\lambda \in\left[\lambda_{-}, \lambda_{+}\right]: f^{\prime}(\infty, \lambda) \in \sigma(-\Delta ; \Omega)\right\}=\left\{\lambda_{0}\right\}
$$

It is clear that under this condition we have

$$
\left\{\lambda \in\left[\lambda_{-}, \lambda_{+}\right]: \nabla_{u}^{2} \Phi(\infty, \lambda) \text { is not an isomorphism }\right\}=\left\{\lambda_{0}\right\} .
$$

We can consider $\operatorname{BIF}\left(\infty,\left[\lambda_{-}, \lambda_{+}\right]\right) \in U(S O(2))$. The following lemma is a direct consequence of Lemma 4.3.1.

Lemma 4.3.2. Let assumption (B.2) holds and let $\lambda_{0}, \lambda_{ \pm} \in \mathbb{R}$ be such that condition (4.3.4) is satisfied. Then $\operatorname{BIF}\left(\infty,\left[\lambda_{-}, \lambda_{+}\right]\right) \neq \Theta \in U(S O(2))$ iff one of the following conditions holds:

(1) $\mathbb{V}_{-\Delta}\left(f^{\prime}\left(\infty, \lambda_{0}\right)\right) \neq \mathbb{V}_{-\Delta}\left(f^{\prime}\left(\infty, \lambda_{0}\right)\right)^{S O(2)}$,

(2) $\mathbb{V}_{-\Delta}\left(f^{\prime}\left(\infty, \lambda_{0}\right)\right)$ is odd.

The next theorem is the consequence of the above lemma and Theorem 2.7

Theorem 4.3.2. Let assumption (B.2) holds and let $\lambda_{0}, \lambda_{ \pm} \in \mathbb{R}$ be such that condition 4.3 .4 is satisfied. Moreover, assume that $\mathbb{V}_{-\Delta}\left(f^{\prime}\left(\infty, \lambda_{0}\right)\right) \neq \mathbb{V}_{-\Delta}\left(f^{\prime}\left(\infty, \lambda_{0}\right)\right)^{S O(2)}$ or $\operatorname{dim} \mathbb{V}_{-\Delta}\left(f^{\prime}\left(\infty, \lambda_{0}\right)\right)$ is odd. Then the statement of Theorem 4.3.1 holds true and moreover $\mathcal{C}$ meets $\left(\infty, \lambda_{0}\right)$.

\section{EXAMPLES}

In this section we illustrate the abstract results proved in the previous section.

Define $\mathbb{V}_{1}=\mathbb{R}[1,1], \mathbb{V}_{2}=\mathbb{V}_{1} \oplus \mathbb{R}[1,0]$ and denote by $\Omega_{1} \subset \mathbb{V}_{1}$ an open disc of radius one in $\mathbb{V}_{1}$ and $\Omega_{2}=\Omega_{1} \times(0,1) \subset \mathbb{V}_{2}$. Since $S O(2)$-representations $\mathbb{V}_{1}, \mathbb{V}_{2}$ are orthogonal, sets $\Omega_{1}, \Omega_{2}$ are $S O(2)$-invariant. First we remind some standard facts about $\sigma\left(-\Delta, \Omega_{i}\right)$, $i=1,2$.

Throughout this section we assume that $k, n \in \mathbb{N} \cup\{0\}$. Moreover, if $k \in \mathbb{N}$, then $n \in \mathbb{N}$ and by $x_{k n}$ we denote the $n$-th solution of $J_{k}^{\prime}(x)=0$ in $(0,+\infty)$, where $J_{k}$ is an $k$-th Bessel function. If $k=0$, then $n \in \mathbb{N} \cup\{0\}$ and by $x_{0 n}$ we denote the $n$-th solution of $J_{0}^{\prime}(x)=0$ in $[0,+\infty)$. Notice that $x_{00}=0$. 
Lemma 5.1. (18]) Under the above assumptions

(1) $\sigma\left(-\Delta, \Omega_{1}\right)=\left\{\lambda_{k n}=x_{k n}^{2}\right\}_{k=1, n=1}^{\infty} \cup\left\{\lambda_{0 n}=x_{0 n}^{2}\right\}_{n=0}^{\infty}$; with corresponding eigenvectors in spherical coordinates given by

(a) if $k>0$, then $n>0$ and $\lambda_{k n} \longrightarrow v_{k n}(r, \phi)=J_{k}\left(x_{k n} r\right)\left\{\begin{array}{l}\cos k \phi, \\ \sin k \phi,\end{array}\right.$

(b) if $k=0$, then $\lambda_{0 n} \longrightarrow v_{0 n}(r, \phi)=J_{0}\left(x_{0 n} r\right)$.

(2) $\sigma\left(-\Delta, \Omega_{2}\right)=\left\{\lambda_{k n j}=(\pi n)^{2}+x_{k j}^{2}\right\}_{k=1, n=0, j=1}^{\infty} \cup\left\{\lambda_{0 n j}=(\pi n)^{2}+x_{0 j}^{2}\right\}_{n=0, j=0}^{\infty}$; with corresponding eigenvectors in cylindrical coordinates given by

(a) if $k>0$, then $j>0$ and $\lambda_{k n j} \longrightarrow v_{k n j}(r, \phi, z)=\cos (n \pi z) J_{k}\left(x_{k j} r\right)\left\{\begin{array}{l}\cos k \phi, \\ \sin k \phi \text {, }\end{array}\right.$

(b) if $k=0$, then $\lambda_{0 n j} \longrightarrow v_{0 n j}(r, \phi, z)=\cos (n \pi z) J_{0}\left(x_{0 j} r\right)$.

In the next lemma we show some properties of zeros of derivatives of Bessel functions.

Lemma 5.2. Under the above assumptions

(1) $0=x_{00}<x_{01}<x_{02}<\ldots$,

(2) $0<x_{k 1}<x_{k 2}<x_{k 3}<\ldots$, for $k \in \mathbb{N}$,

(3) $x_{11}<x_{21}<x_{31}<\ldots$,

Applying Lemmas 5.1, 5.2 we obtain the following corollary.

Corollary 5.1. Under the above assumptions

(1) $\lambda_{00}<\lambda_{01}<\ldots$,

(2) $\lambda_{11}<\lambda_{21}<\ldots$,

(3) $\lambda_{101}<\lambda_{201}<\ldots$

In Lemma 5.3 we describe eigenspaces of $-\Delta$ corresponding to eigenvalues $\sigma\left(-\Delta ; \Omega_{1}\right)$ as $S O(2)$-representations.

Lemma 5.3. If $k \in \mathbb{N} \cup\{0\}, n \in \mathbb{N}$ and $\lambda_{k n} \in \sigma\left(-\Delta ; \Omega_{1}\right)$, then $\mathbb{R}[1, k] \subset \mathbb{V}_{-\Delta}\left(\lambda_{k n}\right)$. Additionally, $\mathbb{V}_{-\Delta}\left(\lambda_{00}\right)=\mathbb{R}[1,0]$.

Proof. First of all notice that from Lemma 5.1 we obtain

(1) if $k>0$, then $\operatorname{span}_{\mathbb{R}}\left\{J_{k}\left(x_{k n} r\right) \cos k \phi, J_{k}\left(x_{k n} r\right) \sin k \phi\right\} \subset \mathbb{V}_{-\Delta}\left(\lambda_{k n}\right)$,

(2) if $k=0$ and $n>0$, then $\operatorname{span}_{\mathbb{R}}\left\{J_{0}\left(x_{0 n} r\right)\right\} \subset \mathbb{V}_{-\Delta}\left(\lambda_{0 n}\right)$,

(3) if $k=0$ and $n=0$, then $\operatorname{span}_{\mathbb{R}}\left\{v_{00}\right\}=\mathbb{V}_{-\Delta}\left(\lambda_{00}\right)$.

Since the $S O(2)$-action $S O(2) \times \mathbb{H}^{1}\left(\Omega_{1}\right) \rightarrow \mathbb{H}^{1}\left(\Omega_{1}\right)$ is given by

$$
\left(\left[\begin{array}{rr}
\cos \theta & -\sin \theta \\
\sin \theta & \cos \theta
\end{array}\right], u\right)(r, \phi)=u(r, \phi+\theta),
$$

it is easy to check that

(1) $\operatorname{span}_{\mathbb{R}}\left\{J_{k}\left(x_{k n} r\right) \cos k \phi, J_{k}\left(x_{k n} r\right) \sin k \phi\right\} \approx \mathbb{R}[1, k]$,

(2) $\operatorname{span}_{\mathbb{R}}\left\{J_{0}\left(x_{0 n} r\right)\right\} \approx \mathbb{R}[1,0]$,

(3) $\operatorname{span}_{\mathbb{R}}\left\{v_{00}\right\} \approx \mathbb{R}[1,0]$,

which completes the proof.

The corollary below is a consequence of Lemma 5.3 
Corollary 5.2. If $\lambda \in \sigma\left(-\Delta, \Omega_{1}\right)$ and

$$
\left\{(k, n) \in(\mathbb{N} \cup\{0\})^{2}: \lambda_{k n} \in \sigma\left(-\Delta, \Omega_{1}\right) \text { and } \lambda_{k n}=\lambda\right\}=\left\{\left(k_{1}, n_{1}\right), \ldots,\left(k_{s}, n_{s}\right)\right\},
$$

then $\mathbb{V}_{-\Delta}(\lambda) \simeq \mathbb{R}\left[1, k_{1}\right] \oplus \mathbb{R}\left[1, k_{2}\right] \oplus \cdots \oplus \mathbb{R}\left[1, k_{s}\right]$.

Moreover, if $a>0$ and $\nu(a)=\sum_{\lambda_{k n}<a} \operatorname{dim} \mathbb{V}_{-\Delta}\left(\lambda_{k n}\right)$, then

$$
\nu(a) \text { is even iff } \#\left\{\lambda_{0 n}: \lambda_{0 n}<a\right\} \text { is even . }
$$

In Lemma 5.4 we describe eigenspaces of $-\Delta$ corresponding to eigenvalues $\sigma\left(-\Delta ; \Omega_{2}\right)$ as $S O(2)$-representations.

Lemma 5.4. If $k, n, j \in \mathbb{N} \cup\{0\}$ and $\lambda_{k n j} \in \sigma\left(-\Delta ; \Omega_{2}\right)$, then $\mathbb{R}[1, k] \subset \mathbb{V}_{-\Delta}\left(\lambda_{k n j}\right)$. Additionally, $\mathbb{V}_{-\Delta}\left(\lambda_{000}\right)=\mathbb{R}[1,0]$.

Proof. In fact the proof is the same as the proof of Lemma 5.3. The details are left to the reader.

The following corollary is a direct consequence of Lemmas 5.4

Corollary 5.3. If $\lambda \in \sigma\left(-\Delta, \Omega_{2}\right)$ and

$\left\{(k, n, j) \in(\mathbb{N} \cup\{0\})^{3}\right\}: \lambda_{k n j} \in \sigma\left(-\Delta, \Omega_{2}\right)$ and $\left.\lambda_{k n j}=\lambda\right\}=\left\{\left(k_{1}, n_{1}, j_{1}\right), \ldots,\left(k_{s}, n_{s}, j_{s}\right)\right\}$, then $\mathbb{V}_{-\Delta}(\lambda) \simeq \mathbb{R}\left[1, k_{1}\right] \oplus \mathbb{R}\left[1, k_{2}\right] \oplus \cdots \oplus \mathbb{R}\left[1, k_{s}\right]$.

Moreover, if $a>0$ and $\nu(a)=\sum_{\lambda_{k n j}<a} \operatorname{dim} \mathbb{V}_{-\Delta}\left(\lambda_{k n j}\right)$, then

$$
\nu(a) \text { is even iff } \#\left\{\lambda_{0 n j}: \lambda_{0 n j}<a\right\} \text { is even . }
$$

Remark 5.1. For $i=1,2$ let $\lambda_{0}\left(\Omega_{i}\right)$ be the smallest eigenvalue in $\sigma\left(-\Delta, \Omega_{i}\right)$, such that $\mathbb{V}_{-\Delta}\left(\lambda_{0}\left(\Omega_{i}\right)\right)$ is a nontrivial $S O(2)$-representation. It clear that

$$
\lambda_{0}\left(\Omega_{1}\right)=\lambda_{11}=x_{11}^{2}=\lambda_{101}=\lambda_{0}\left(\Omega_{2}\right) .
$$

The proof of the lemma below is a direct consequence of estimations from [32, Section 15.3, p.486].

Lemma 5.5. For every $k \in \mathbb{N}, \lambda_{k 1} \in \sigma\left(-\Delta, \Omega_{1}\right)$ and $\lambda_{k 01} \in \sigma\left(-\Delta, \Omega_{2}\right)$ we have

$$
k(k+2)<\lambda_{k 1}<2 k(k+1), \quad k(k+2)<\lambda_{k 01}<2 k(k+1) .
$$

Consequently, $3<\lambda_{0}\left(\Omega_{1}\right)=\lambda_{0}\left(\Omega_{2}\right)<4$.

Example 5.1. Consider equation

$$
\left\{\begin{aligned}
-\Delta u & =f(u) & & \text { in } \Omega_{1} \\
\frac{\partial u}{\partial \nu} & =0 & & \text { on } \partial \Omega_{1},
\end{aligned}\right.
$$

where $f$ satisfies the following assumptions:

(1) $f \in C^{1}(\mathbb{R}, \mathbb{R})$,

(2) $\left|f^{\prime}(x)\right| \leq a+b|x|^{q}$ for some $a, b>0, q \in \mathbb{N}$,

(3) $f(t)=f^{\prime}(\infty) t+o(|t|)$, where $|t| \rightarrow \infty$, 
(4) $50<f^{\prime}(\infty)<99$,

(5) $\# Z<\infty$, where $Z=f^{-1}(0)$,

(6) $f^{\prime}(z) \notin \sigma\left(-\Delta, \Omega_{1}\right)$, for all $z \in Z$,

(7) there are $z_{0}, z_{1} \in Z$ such that $4<f^{\prime}\left(z_{1}\right)<99<f^{\prime}\left(z_{0}\right)$.

It is clear that $f$ satisfies assumptions (A.1)-(A.5) of the previous section. Moreover, it is known that $\lambda_{02}=x_{02}^{2} \approx 49<f^{\prime}(\infty)<100 \approx x_{03}^{2}=\lambda_{03}$. Therefore by Corollary 5.2 we obtain that $\nu\left(f^{\prime}(\infty)\right)$ is odd.

Taking into account assumption (7) and Lemma 5.5 we obtain that $f^{\prime}\left(z_{0}\right)>f^{\prime}\left(z_{1}\right)>$ $\lambda_{0}\left(\Omega_{1}\right)$ and $f^{\prime}\left(z_{0}\right)>f^{\prime}(\infty)$. Now it is easy to verify that under the above assumptions $f$ satisfies assumption (1) of Theorem 4.1.3. Thus there exists at least one nonconstant weak solution of equation (5.1).

If there exists exactly one $z_{0} \in Z$ such that $f^{\prime}\left(z_{0}\right)>\lambda_{0}\left(\Omega_{1}\right)$, then in order to use Theorem 4.1.3 we have to replace assumption (71) with the following assumption:

$\left(7^{\prime}\right)$ there exists $k^{\prime}, n^{\prime} \in \mathbb{N}$ such that $f^{\prime}\left(z_{0}\right)<\lambda_{k^{\prime} n^{\prime}}<50\left(99<\lambda_{k^{\prime} n^{\prime}}<f^{\prime}\left(z_{0}\right)\right)$.

Indeed, with assumption (7) replaced by assumption (7') the assumption (2) of Theorem 4.1 .3 is fulfilled.

Example 5.2. Consider equation

$$
\left\{\begin{aligned}
-\Delta u & =f(u) & & \text { in } \Omega_{2}, \\
\frac{\partial u}{\partial \nu} & =0 & & \text { on } \partial \Omega_{2},
\end{aligned}\right.
$$

where $f$ satisfies the following assumptions:

(1) $f \in C^{1}(\mathbb{R}, \mathbb{R})$,

(2) $\left|f^{\prime}(x)\right| \leq a+b|x|^{3}$ for some $a, b>0$,

(3) $f(t)=f^{\prime}(\infty) t+o(|t|)$, where $|t| \rightarrow \infty$,

(4) $f^{\prime}(\infty)<0$

(5) $\# Z<\infty$, where $Z=f^{-1}(0)$,

(6) $f^{\prime}(z) \notin \sigma\left(-\Delta, \Omega_{2}\right)$, for all $z \in Z$,

(7) there exists $z_{0} \in Z$ such that $f^{\prime}\left(z_{0}\right)>4$.

It is clear that $f$ satisfies assumptions (A.1)-(A.5) of the previous section. Moreover, by assumption (7) and Lemma 5.5, $f^{\prime}\left(z_{0}\right)>\lambda_{0}\left(\Omega_{2}\right)$. Applying Theorem 4.1.2 we obtain nonconstant weak solutions of equation (5.2).

Define $Z_{+}=\left\{z \in Z \mid f^{\prime}(z)>0\right\}$ and assume that $0<f^{\prime}(z)<9$, for every $z \in Z_{+}$. Since $x_{01} \simeq 3.83, f^{\prime}(z)<\lambda_{001}=x_{01}^{2}$ and $f^{\prime}(z)<\lambda_{010}=\pi^{2}$. By Lemmas [5.1] 5.2] we obtain $\left\{\lambda_{0 n j} \in \sigma\left(-\Delta, \Omega_{2}\right): \lambda_{0 n j}<9\right\}=\left\{\lambda_{000}\right\}$. Therefore, by Corollary 5.2, we obtain that $\nu\left(f^{\prime}(z)\right)$ is odd for every $z \in Z_{+}$. Notice that assumptions of Theorem 4.1.1 are not fulfilled. In other words we can not apply the Leray-Schauder degree to obtain the existence of nonconstant weak solutions of equation (5.2).

Example 5.3. Consider equation (5.2) and assume that

(1) $f \in C^{1}(\mathbb{R}, \mathbb{R})$,

(2) $\left|f^{\prime}(x)\right| \leq a+b|x|^{3}$ for some $a, b>0$,

(3) $f(t)=f^{\prime}(\infty) t+o(|t|)$, where $|t| \rightarrow \infty$, 
(4) $\# Z<\infty$,

(5) there exists $z_{0} \in Z$ and $k^{\prime} \in \mathbb{N}$ such that:

(a) $f^{\prime}\left(z_{0}\right) \notin \sigma\left(-\Delta, \Omega_{2}\right)$,

(b) $f^{\prime}\left(z_{0}\right)>2 k^{\prime}\left(k^{\prime}+1\right)$,

(c) $f^{\prime}(z)<k^{\prime}\left(k^{\prime}+2\right)$ for $z \in(Z \cup\{\infty\}) \backslash\left\{z_{0}\right\}$.

It is clear that $f$ satisfies assumptions (A.1), (A.2) and (A.3) of the previous section. Combining assumptions (5.b), (5.c) with Lemma 5.5 we obtain that $f^{\prime}(z)<\lambda_{k^{\prime} 01}<f^{\prime}\left(z_{0}\right)$ for all $z \in(Z \cup\{\infty\}) \backslash\left\{z_{0}\right\}$. Fix $\lambda \in \sigma\left(-\Delta, \Omega_{1}\right) \cap\left(0, \lambda_{k^{\prime} 01}\right)$. By Corollary 5.3, $\mathbb{V}_{-\Delta}(\lambda) \simeq$ $\mathbb{R}\left[1, k_{1}\right] \oplus \ldots \oplus \mathbb{R}\left[1, k_{s}\right]$ for some $k_{1}, \ldots, k_{s} \in \mathbb{N} \cup\{0\}$.

We claim that $k_{i}<k^{\prime}$ for every $1 \leq i \leq s$. Suppose, contrary to our claim, that $k_{i_{0}} \geq k^{\prime}$ for some $1 \leq i_{0} \leq s$. Then, by Corollary [5.3, there exist $n_{i_{0}} \in \mathbb{N} \cup\{0\}, j_{i_{0}} \in \mathbb{N}$ such that $\lambda_{k_{i_{0}} n_{i_{0}} j_{i_{0}}}=\lambda$. From Lemmas [5.1, 5.2 we obtain

$$
\lambda_{k_{i_{0}} n_{i_{0}} j_{i_{0}}}=\left(\pi n_{i_{0}}\right)^{2}+x_{k_{i_{0}} j_{i_{0}}}^{2} \geq x_{k_{i_{0}} j_{i_{0}}}^{2} \geq x_{k_{i_{0}} 1}^{2}=\lambda_{k_{i_{0}} 01} \text {. }
$$

By Corollary [5.1] we obtain $\lambda=\lambda_{k_{i_{0}} n_{i_{0}} j_{i_{0}}} \geq \lambda_{k_{i_{0}} 01} \geq \lambda_{k^{\prime} 01}$, a contradiction. Thus $k_{i}<k^{\prime}$ for $i=1, \ldots, s$ and consequently $\mathbb{V}(\lambda)_{\mathbb{Z}_{k^{\prime}}}=\emptyset$. Taking into account assumption (5.c) and Lemma 5.5 we obtain $\mathbb{V}_{-\Delta}\left(f^{\prime}(z)\right)_{\mathbb{Z}_{k^{\prime}}}=\emptyset$ for all $z \in(Z \cup\{\infty\}) \backslash\left\{z_{0}\right\}$ such that $f^{\prime}(z) \in \sigma\left(-\Delta, \Omega_{2}\right)$.

Notice that all the assumptions of Theorem 4.1.5 are satisfied. Applying this theorem we obtain the existence of at least one nonconstant weak solutions of equation (5.2).

Suppose now that (5.a) does not hold, i.e. $f^{\prime}\left(z_{0}\right) \in \sigma\left(-\Delta, \Omega_{2}\right)$. In order to obtain the existence of weak nonconstant solutions of equation (5.2) we have to assume:

(5.a') $\mathbb{V}_{-\Delta}\left(f^{\prime}\left(z_{0}\right)\right)^{S O(2)}=\{0\}$ and $\mathbb{V}_{-\Delta}\left(f^{\prime}\left(z_{0}\right)\right)_{\mathbb{Z}_{k^{\prime}}}=\emptyset$.

It is clear that under the above assumption and assumptions (1)-(3), (5.b) and (5.c) Theorem 4.1.5 holds. This assumption is equivalent to the following one

(5.a") $f^{\prime}\left(z_{0}\right) \neq \lambda_{0 n j}$ for $n, j \in \mathbb{N} \cup\{0\}$ and $f^{\prime}\left(z_{0}\right) \neq \lambda_{k^{\prime \prime} n j}$ where $k^{\prime \prime}=k^{\prime} m$ for $m \in \mathbb{N}$, $n \in \mathbb{N} \cup\{0\}, j \in \mathbb{N}$.

Example 5.4. In this example we illustrate bifurcations from infinity. Consider the family of equations

$$
\left\{\begin{aligned}
-\Delta u & =f(u, \lambda) & & \text { in } \Omega_{1} \\
\frac{\partial u}{\partial \nu} & =0 & & \text { on } \partial \Omega_{1},
\end{aligned}\right.
$$

where $f$ satisfies the following assumptions:

(1) $f \in C^{1}(\mathbb{R} \times \mathbb{R}, \mathbb{R})$

(2) $\left|f^{\prime}(x, \lambda)\right| \leq a+b|x|^{q}$ for some $a, b>0, q \in \mathbb{N}$ and all $\lambda \in \mathbb{R}$,

(3) there exist limits $f^{\prime}\left(\infty, \lambda_{ \pm}\right)$for some $\lambda_{+}, \lambda_{-}>0$,

(4) $f^{\prime}\left(\infty, \lambda_{ \pm}\right) \notin \sigma\left(-\Delta, \Omega_{1}\right)$,

(5) $f^{\prime}\left(\infty, \lambda_{-}\right)<f^{\prime}\left(\infty, \lambda_{+}\right)$.

Under the above assumptions $\operatorname{BIF}\left(\infty,\left[\lambda_{-}, \lambda_{+}\right]\right) \neq \Theta \in U(S O(2))$ iff one of the following conditions is satisfied:

(a) there exists $\lambda_{k n} \in \sigma\left(-\Delta, \Omega_{1}\right), n, k \in \mathbb{N}$ such that $f^{\prime}\left(\infty, \lambda_{+}\right)>\lambda_{n k}>f^{\prime}\left(\infty, \lambda_{-}\right)$,

(b) $\#\left\{\lambda_{0 n} \in \sigma\left(-\Delta, \Omega_{1}\right): f^{\prime}\left(\infty, \lambda_{-}\right)<\lambda_{0 n}<f^{\prime}\left(\infty, \lambda_{+}\right)\right\}$is odd. 
Therefore, all the assumptions of Theorem 4.3.1 are fulfilled if one of the conditions (a), (b) is satisfied.

Let us now replace condition (3) with the following:

(3') there exists limit $f^{\prime}(\infty, \lambda)$, for all $\lambda \in \mathbb{R}$.

Let $\lambda_{0}$ be such that $f^{\prime}\left(\infty, \lambda_{0}\right) \in \sigma(-\Delta ; \Omega)$. If there exists $k, n \in \mathbb{N}$ such that $f^{\prime}\left(\infty, \lambda_{0}\right)=$ $\lambda_{k n}$, then by Corollary $\left[5.2\right.$ we get $\mathbb{V}_{-\Delta}\left(f^{\prime}\left(\infty, \lambda_{0}\right)\right) \neq \mathbb{V}_{-\Delta}\left(f^{\prime}\left(\infty, \lambda_{0}\right)\right)^{S O(2)}$. Otherwise, $f^{\prime}\left(\infty, \lambda_{0}\right)=\lambda_{0 n}$ for some $n \in \mathbb{N}$ and from Corollaries [5.1] and [5.2 we conclude that $\operatorname{dim} \mathbb{V}_{-\Delta}\left(f^{\prime}\left(\infty, \lambda_{0}\right)\right)=1$. Hence, if there exists $\lambda_{-}<\lambda_{+}$such that

$$
\left\{\lambda \in\left[\lambda_{-}, \lambda_{+}\right]: f^{\prime}(\infty, \lambda) \in \sigma(-\Delta ; \Omega)\right\}=\left\{\lambda_{0}\right\},
$$

then $\operatorname{BIF}\left(\infty,\left[\lambda_{-}, \lambda_{+}\right]\right) \neq \Theta \in U(S O(2))$. Applying Theorem 4.3.2 we obtain the existence of an unbounded connected set $\mathcal{C}$ of weak solutions of equation (5.3) which meets $\left(\infty, \lambda_{0}\right)$.

Now let $f(x, \lambda)=\lambda f(u)$ and assume that $f^{\prime}(\infty)=f^{\prime}(\infty, 1) \neq 0$. Fix $\lambda_{i_{0}} \in \sigma\left(-\Delta, \Omega_{1}\right)$ and put $\lambda_{0}=\frac{\lambda_{i_{0}}}{f^{\prime}(\infty)}$. Then there exists $\epsilon>0$ and $\lambda_{ \pm}=\lambda_{0} \pm \epsilon$ such

$$
\left\{\lambda \in\left[\lambda_{-}, \lambda_{+}\right]: \lambda f^{\prime}(\infty) \in \sigma(-\Delta ; \Omega)\right\}=\left\{\lambda_{0}\right\} .
$$

Consequently, $\operatorname{BIF}\left(\infty,\left[\lambda_{-}, \lambda_{+}\right]\right) \neq \Theta \in U(S O(2))$. By Theorem 4.3.2 there exists an unbounded connected set $\mathcal{C}$ of weak solutions of equation (5.3) which meets $\left(\infty, \lambda_{0}\right)$.

\section{REFERENCES}

[1] J. F. Adams, Lectures on Lie groups, W. A. Benjamin Inc., New York-Amsterdam, (1969),

[2] A. Ambrosetti, Branching points for a class of variational operators, J. Anal. Math. 76 (1998), 321-335,

[3] R. Böhme, Die Lösung der versweigungsgleichungen für nichtlineare eigenwert-probleme, Math. Z. 127 (1972), 105-126,

[4] J. Byeon, Singularly perturbed nonlinear Neumann problems on symmetric domains., Comm. Partial Diff. Equat. 26(9-10) (2001), 1607-1631,

[5] E.N. Dancer \& S. Yan, Solutions with interior and boundary peaks for the Neumann problem of an elliptic system of FitzHugh-Nagumo type, Indiana Univ. Math. J. 55(1) (2006), 217-258,

[6] E. N. Dancer \& S. Yan, Multipeak solutions for the Neumann problem of an elliptic system of FitzHugh-Nagumo type, Proc. London Math. Soc. (3) 90(1) (2005), 209-244,

[7] E. N. Dancer \& S. Yan, Multipeak solutions for a singularly perturbed Neumann problem, Pacific J. Math. 189(2) (1999), 241-262,

[8] T. tom Dieck, Transformation groups, Walter de Gruyter, Berlin-New York, 1987,

[9] J. Fura, A. Ratajczak \& S. Rybicki, Existence and continuation of periodic solutions of Newtonian systems, J. Diff. Equat. 218(1) (2005), 216-252,

[10] J. Fura \& S. Rybicki, Bifurcation from infinity of periodic solutions of second order Hamiltonian systems, to appear in Ann. de l'Inst. Henri Poincaré Anal. non Lin., (2006),

[11] M. Grossi, A. Pistoia \& J. Wei, Existence of multipeak solutions for a semilinear Neumann problem via nonsmooth critical point theory. Calc. Var. Partial Differential Equations 11(2) (2000), 143-175,

[12] N. Hirano \& K. Wan, Multiple existence of solutions for a semilinear elliptic problem with Neumann boundary condition, J. Math. Anal. Appl. 314(1) (2006), 210-218,

[13] J. Ize, Topological bifurcation, Topological Nonlinear Analysis, Degree, Singularity and Variations, Eds. M. Matzeu i A. Vignoli, Progr. Nonlinear Differential Equations Appl. 15, Birkhäuser, (1995), $341-463$ 
[14] B. Ko, The existence of nonconstant solutions of a class of semilinear elliptic Neumann problems with a small parameter, Nonl. Anal. TMA 28(7) (1997), 1249-1263,

[15] C. Li, The existence of infinitely many solutions of a class of nonlinear elliptic equations with Neumann boundary condition for both resonance and oscillation problems, Nonl. Anal. TMA 54(3) (2003), 431-443,

[16] C. Li \& S. Li, Multiple solutions and sign-changing solutions of a class of nonlinear elliptic equations with Neumann boundary condition, J. Math. Anal. Appl. 298(1) (2004), 14-32,

[17] A. Marino, La biforcazione nel caso variazionale, Conf. Sem. Mat. Univ. Bari 132 (1977),

[18] C.W. Michlin, Linear equations of mathematical physics, Science, Moscow, (1964), (in Russian),

[19] A. Pomponio, A multiplicity result for solutions of a nonlinear elliptic system with Neumann conditions, Red. di Mat. (VII) (22) (2002), 377-391,

[20] P. Rabier Symmetries, topological degree and a theorem of Z. Q. Wang, Rocky Mount. J. of Math. 24(3) (1994), 1087-1115,

[21] S. Rybicki, $S O(2)$-degree for orthogonal maps and its applications to bifurcation theory, Nonl. Anal. TMA 23(1) (1994), 83-102,

[22] S. Rybicki, Applications of degree for $S O(2)$-equivariant gradient maps to variational nonlinear problems with SO(2)-symmetries, Topol. Meth. Nonl. Anal. 9(2) (1997), 383-417,

[23] F. Takens, Some remarks on the Böhme-Berger bifurcation theorem, Math. Z. 125 (1972), 359-364,

[24] C. L. Tang, Multiple solutions of Neumann problem for elliptic equations, Nonl. Anal. 54(4) (2003), 637-650,

[25] C. L. Tang \& X. P. Wu, Existence and nultiplicity for solutions of Neumann problem for semilinear elliptic equations, J. Math Anal. Appl. 288(2) (2003), 660-670,

[26] C. L. Tang \& X. P. Wu, Multiple solutions of a class of Neumann problem for semilinear elliptic equations, Nonlinear Anal. 62(3) (2005), 455-465,

[27] G. Vannella, Existence and multiplicity of solutions for a nonlinear Neumann problem, Ann. Mat. Pura Appl. 180(4) (2002), 429-440,

[28] G. Vannella, Multiplicity results for two kinds of equivariant systems, Nonl. Anal. TMA 59(3) (2004), 283-304,

[29] Z. Q. Wang, Existence and nonexistence of G-least energy solutions for a nonlinear Neumann problem with critical exponent in symmetric domains, Calc. Var. Partial Diff. Equat. 8(2) (1999), 109-122,

[30] Z. Q. Wang, Construction of multi-peaked solutions for a nonlinear Neumann problem with critical exponent in symmetric domains, Nonl. Anal. TMA 27(11) (1996), 1281-1306,

[31] Z. Q. Wang, Nonradial solutions of nonlinear Neumann problems in radially symmetric domains, Topology in nonlinear analysis (Warsaw, 1994), 85-96,

[32] G. N. Watson A treatise on the theory of Bessel functions, Cambrige University Press, London and New York, (1944),

[33] S. Yan, On the number of interior multipeak solutions for singularly perturbed Neumann problems, Topol. Meth. Nonl. Anal. 12(1) (1998), 61-78.

Faculty of Mathematics and Computer Science, Nicolaus Copernicus University, PL87-100 Toruń, ul. Chopina 12/18, Poland

E-mail address: Krzysztof.Muchewicz@mat.uni.torun.pl

Faculty of Mathematics and Computer Science, Nicolaus Copernicus University, PL87-100 Toruń, ul. Chopina 12/18, Poland

E-mail address: Slawomir.Rybicki@mat.uni.torun.pl 